\title{
Evolutionarily Novel Functional Networks in the Human Brain?
}

\author{
Dante Mantini, ${ }^{1,2,3}$ Maurizio Corbetta, ${ }^{2,3,4,5}$ Gian Luca Romani, ${ }^{2,3}$ Guy A. Orban, ${ }^{1,6}$ and Wim Vanduffel ${ }^{1,7,8}$ \\ ${ }^{1}$ Laboratory of Neuro- and Psychophysiology, KU Leuven Medical School, Leuven, 3000, Belgium, ${ }^{2}$ Department of Neuroscience and Imaging, D’Annunzio \\ University, Chieti, 66013, Italy, ${ }^{3}$ Institute for Advanced Biomedical Technology, D’Annunzio University Foundation, Chieti, 66013, Italy, Departments of \\ ${ }^{4}$ Neurology and ${ }^{5}$ Radiology, Washington University, St. Louis, Missouri 63110, ${ }^{6}$ Department of Neuroscience, University of Parma Medical School, Parma, \\ 43100, Italy, ${ }^{7}$ Athinoula A. Martinos Center for Biomedical Imaging, Charlestown, Massachusetts 02129, and ${ }^{8}$ Department of Radiology, Harvard Medical \\ School, Boston, Massachusetts 02115
}

Primate evolution has been accompanied by complex reorganizations in brain anatomy and function. Little is known, however, about the relationship between anatomical and functional changes induced through primate evolution. Using functional magnetic resonance imaging, we assessed spatial and temporal correspondences of cortical networks in humans and monkeys. We provided evidence for topologically and functionally correspondent networks in sensory-motor and attention regions. More specifically, we revealed a possible monkey equivalent of the human ventral attention network. For other human networks, such as the language and the default-mode networks, we detected topological correspondent networks in the monkey, but with different functional signatures. Furthermore, we observed two lateralized human frontoparietal networks in the cortical regions displaying the greatest evolutionary expansion, having neither topological nor functional monkey correspondents. This finding may indicate that these two human networks are evolutionarily novel. Thus, our findings confirm the existence of networks where evolution has conserved both topology and function but also suggest that functions of structurally preserved networks can diverge over time and that novel, hence human-specific networks, have emerged during human evolution.

\section{Introduction}

Humans are characterized by specific cognitive skills, related to intelligence and their ability to manipulate the environment, which distinguish them from all other primates. In searching for correlates of these human-specific abilities, numerous studies have investigated evolution-driven changes in the primate brain. A fascinating, but yet to be answered question is whether humanspecific cognitive abilities emerged from evolutionarily novel human-specific cortical networks. Here we used functional magnetic resonance imaging (fMRI) in awake monkeys to address this question.

Comparative analyses from several species suggest that an increase in brain size during evolution is accompanied by changes in relative size of cortical regions, and modifications in micro-

Received Sept. 14, 2012; revised Nov. 13, 2012; accepted Nov. 27, 2012.

Author contributions: M.C., G.L.R., G.A.O., and W.V. designed research; D.M. performed research; D.M. contributed unpublished reagents/analytic tools; D.M. analyzed data; D.M. and W.V. wrote the paper.

This work received support from European Union Seventh Framework Programme FWP-200728; Inter-University Attraction Pole 6/29; Programme Financing PFV/10/008; Geconcerteerde Onderzoeks Actie 10/19; Impulsfinanciering Zware Apparatuur and Hercules funding of the Katholieke Universiteit Leuven; Fonds Wetenschappelijk Onderzoek-Vlaanderen G062208N10, G083111N10, and G043912N; and Geneeskundige Stichting Koningin Elisabeth prize "Janine en Jacques Delaruelle." The Martinos Center for Biomedical Imaging is supported by National Center for Research Resources grant P41RR14075. We thank C. Fransen, C. Van Eupen, and A. Coeman for animal training and care; J.T. Arsenault, K. Nelissen, 0. Joly, H. Kolster, W. Depuydt, G. Meulemans, P. Kayenbergh, M. De Paep, M. Docx, and I. Puttemans for technical assistance; and S. Raiguel for his comments on this manuscript.

Correspondence should be addressed to Dr. Wim Vanduffel, Athinoula A. Martinos Center for Biomedical Imaging, 13th Street, Building 149, Charlestown, MA 02129. E-mail: wim@nmr.mgh.harvard.edu.

DOI:10.1523/JNEUROSCI.4392-12.2013

Copyright $\odot 2013$ the authors $\quad 0270-6474 / 13 / 333259-17 \$ 15.00 / 0$ structure and connections (Krubitzer, 2007; Sherwood et al., 2008; Allman et al., 2010; Kaas, 2012). Notably, not all brain regions are equally affected by such evolution-driven changes. For instance, the degree of cortical surface expansion in humans (Rakic, 1995) is topologically inhomogeneous, and much more pronounced in associative than in sensory-motor regions (Van Essen and Dierker, 2007). Accordingly, large local increases in the cortical sheet size might account for the development of novel networks in humans, most likely those devoted to novel cognitive abilities (Van Essen and Dierker, 2007; Van Essen et al., 2012).

Hypothetically, one alternative possibility is that this "novel" anatomical territory contains existing cortical networks that simply expanded during evolution. If so, the emergence of novel functions must be related to the functional redeployment of pre-existing brain circuitry during evolution (Anderson, 2010; Fitch, 2011). Functional redeployment, however, may take place not only in cortex with a significantly increased surface area, but in structurally conserved cortex as well. Finally, one should also consider that existing functions may reside in stable, structurally relatively unchanged networks, or may have shifted to pre-existing networks that do not correspond topologically (Mantini et al., 2012b). These possible scenarios for evolution-driven cerebral reorganizations may not be mutually exclusive. Little experimental evidence is currently available, which would allow us to distinguish between cortical networks that are evolutionarily novel, functionally redeployed, or evolutionarily conserved. 


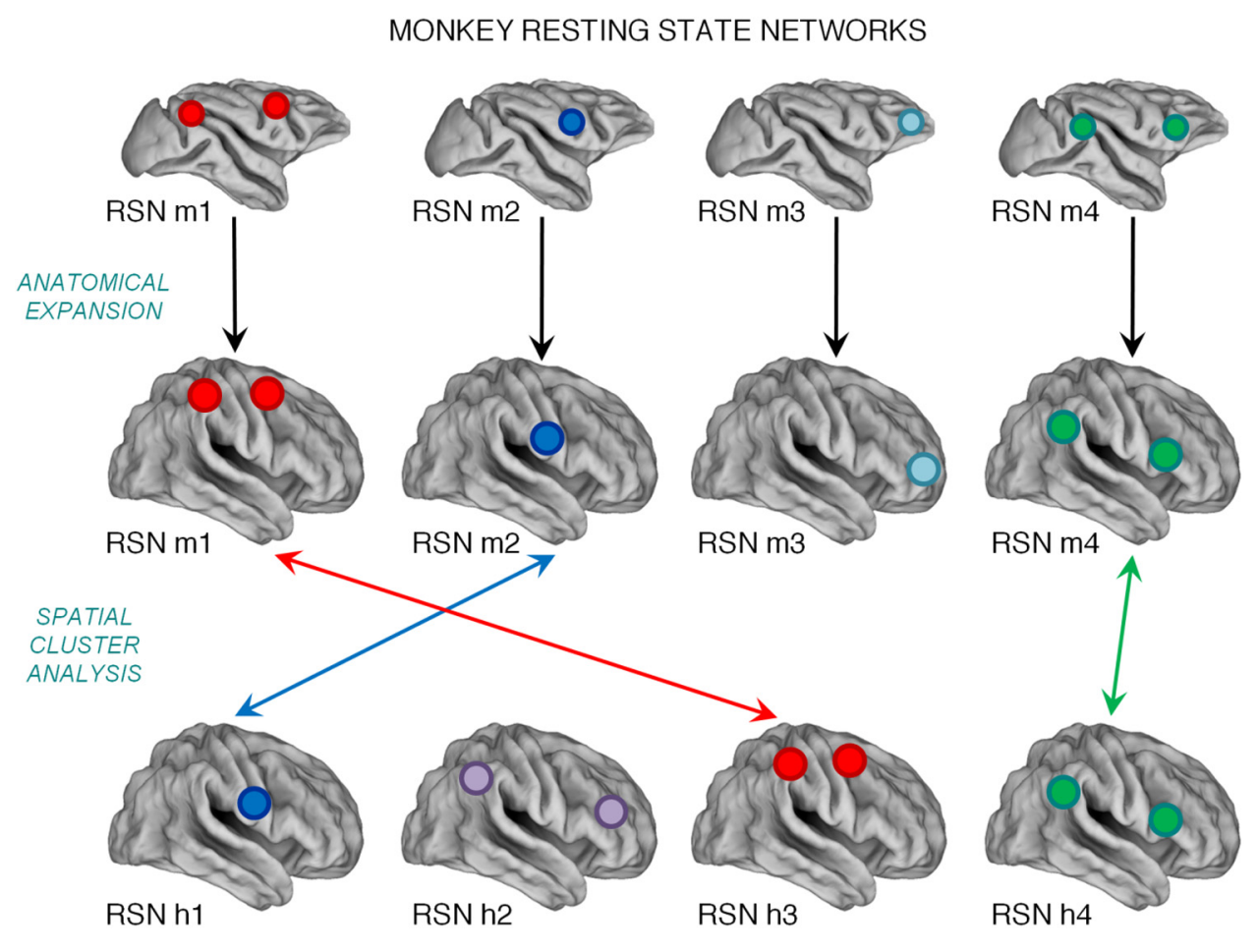

HUMAN RESTING STATE NETWORKS

Figure 1. Method used to identify topologically correspondent RSNs between species. First, the spatial maps of the monkey RSNs were expanded to the human space by means of a landmarkbased model (Van Essen and Dierker, 2007). Cluster analysis was then jointly applied to the monkey and human RSNs, using spatial correlation as a similarity metric. The similar monkey and human RSNs were placed together in the same cluster. Other RSNs that had no correspondent in the other species were fit into single-element clusters.

Our intent was to examine to which degree and which cortical networks may have undergone evolution-driven reorganization. Therefore, we collected fMRI data and applied an analytical strategy through which we assessed both topological and functional similarities between brain networks in two primate species. We performed our analysis in two steps, using restingstate and natural-vision data collected in macaques and humans. First, we used independent-component analysis (ICA) on resting-state data to define networks of brain areas showing correlated task-independent activity (resting-state networks, RSNs). After warping the monkey RSNs to human space, we performed a cluster analysis to establish topological correspondences between human and monkey RSNs. In a second step, we analyzed the functional responses of RSNs in the two species. To this end, we used natural-vision data, since the richness and complexity of the sensory stimulation activates large portions of the cortex (Hasson et al., 2004; Mantini et al., $2012 \mathrm{~b}$ ). By assessing the similarity in the response profiles of the RSNs of the two species, we examined which human RSNs have putative functional correspondents in monkeys.

\section{Materials and Methods}

Subjects. Four rhesus monkeys (Macaca mulatta, one female, 4-6 kg, 4-7 years old) and 24 right-handed young, healthy Italian-speaking volunteers ( 15 females, $20-31$ years old) participated in the study. Animal care procedures met all Belgian and European guidelines, and were approved by the KU Leuven Medical School. Human volunteers were informed about the experimental procedures and signed a written informed consent. The study design was approved by the local Ethics Committees of both the KU Leuven and the Chieti University for experiments using monkeys and humans, respectively. Details on the procedures for the health and welfare of the animals are described in our previous study (Mantini et al., 2012b).
Experiments. We conducted two experiments with the monkey and human subjects, referred to, respectively, as follows: (1) resting state and (2) natural vision. The resting-state data have not been used in any prior study. In two earlier studies (Mantini et al., 2012a,b) we analyzed the monkey and human natural vision fMRI data presented here, but to address a completely different investigative question, namely how to compare, across species, large-scale functional networks activated by large-scale visual and auditory stimulation. In the present study, we first identified cortical networks independently of activations that might be observed during any kind of sensory stimulation. Specifically, we aimed to study the topological similarities of the so-called RSNs in the two primate species (Fox and Raichle, 2007; Smith et al., 2009; Hutchison et al., 2011). Next, using the analytical methods developed by Mantini et al. (2012a,b), we tested whether the identified RSNs in the two primate species showed similar response properties during natural vision.

In the resting-state experiment, the participants had to continuously fixate a point with no visual stimuli present in the background. The reason for introducing a fixation task into a resting-state paradigm is because monkeys need to be motivated by rewards to remain as motionless as possible during data collection. In the natural-vision experiment, the subjects watched and listened to $30 \mathrm{~min}$ of the Italian version of the movie "The Good the Bad and the Ugly" directed by Sergio Leone, from minute 16:48 to minute $46: 48$. The movie was divided into three clips of 10 min each. The movie clips were presented six times to the monkeys, and once to the human subjects.

Procedures. Monkeys were first prepared and trained on this fixation task in a mock-up apparatus mimicking the MR scanner (Vanduffel et al., 2001). Before fMRI scanning, a bolus of microcrystalline iron-oxide nanoparticles (Sinerem; Guerbet; $6-10 \mathrm{mg} / \mathrm{kg}$ ) was injected into the femoral or saphenous vein of the animals to increase the contrast-tonoise ratio and the spatial selectivity relative to the blood oxygen leveldependent (BOLD) technique. Inside the MR scanner, monkeys sat in a sphinx position with their heads fixed to the chair within the horizontal 


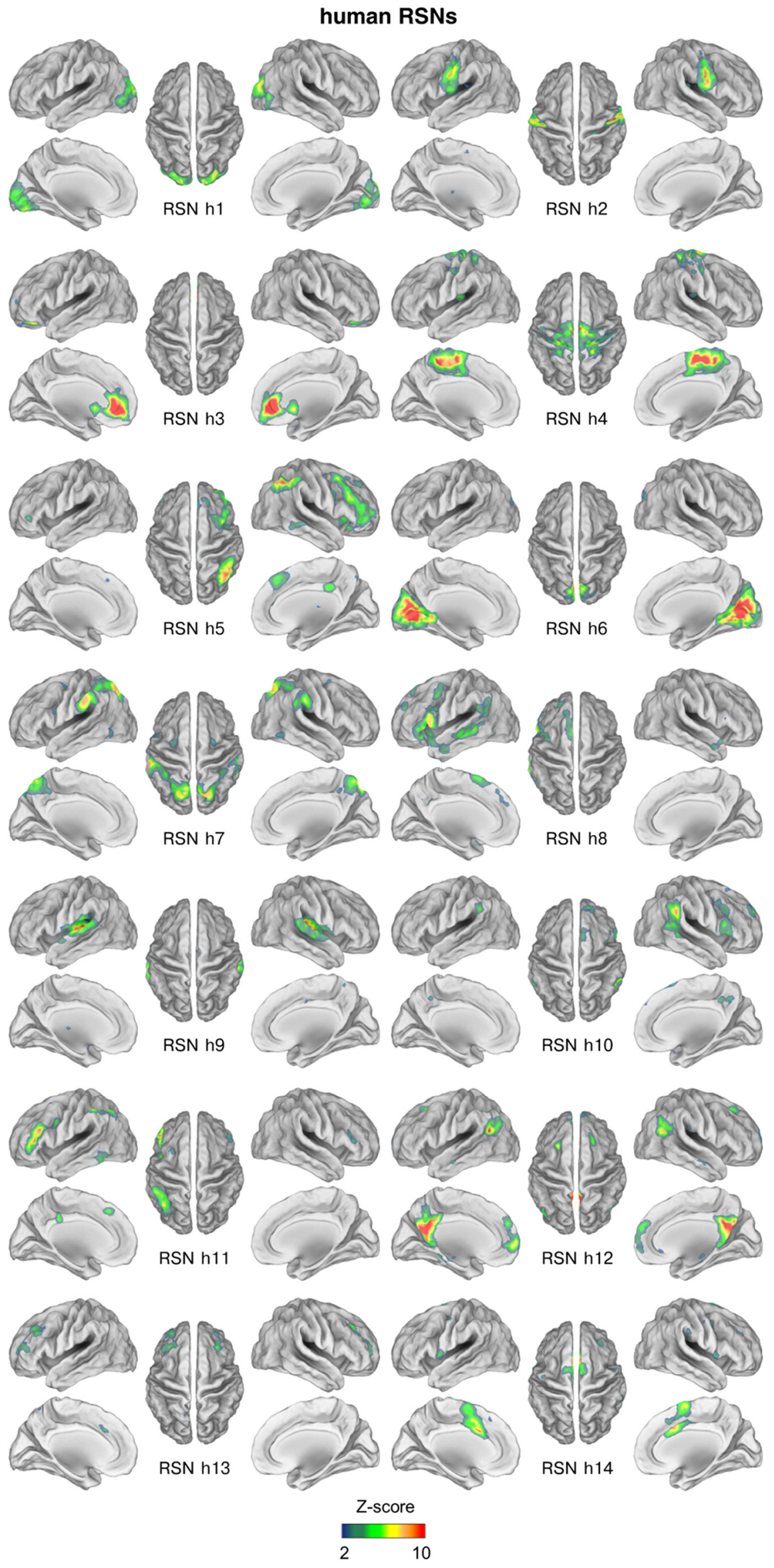

Figure 2. Folded cortical maps of the RSNs detected in humans. Each RSN is plotted (threshold at $Z>2$ ) over a representative cortical surface in dorsal, lateral, and medial views. 
monkey RSNs
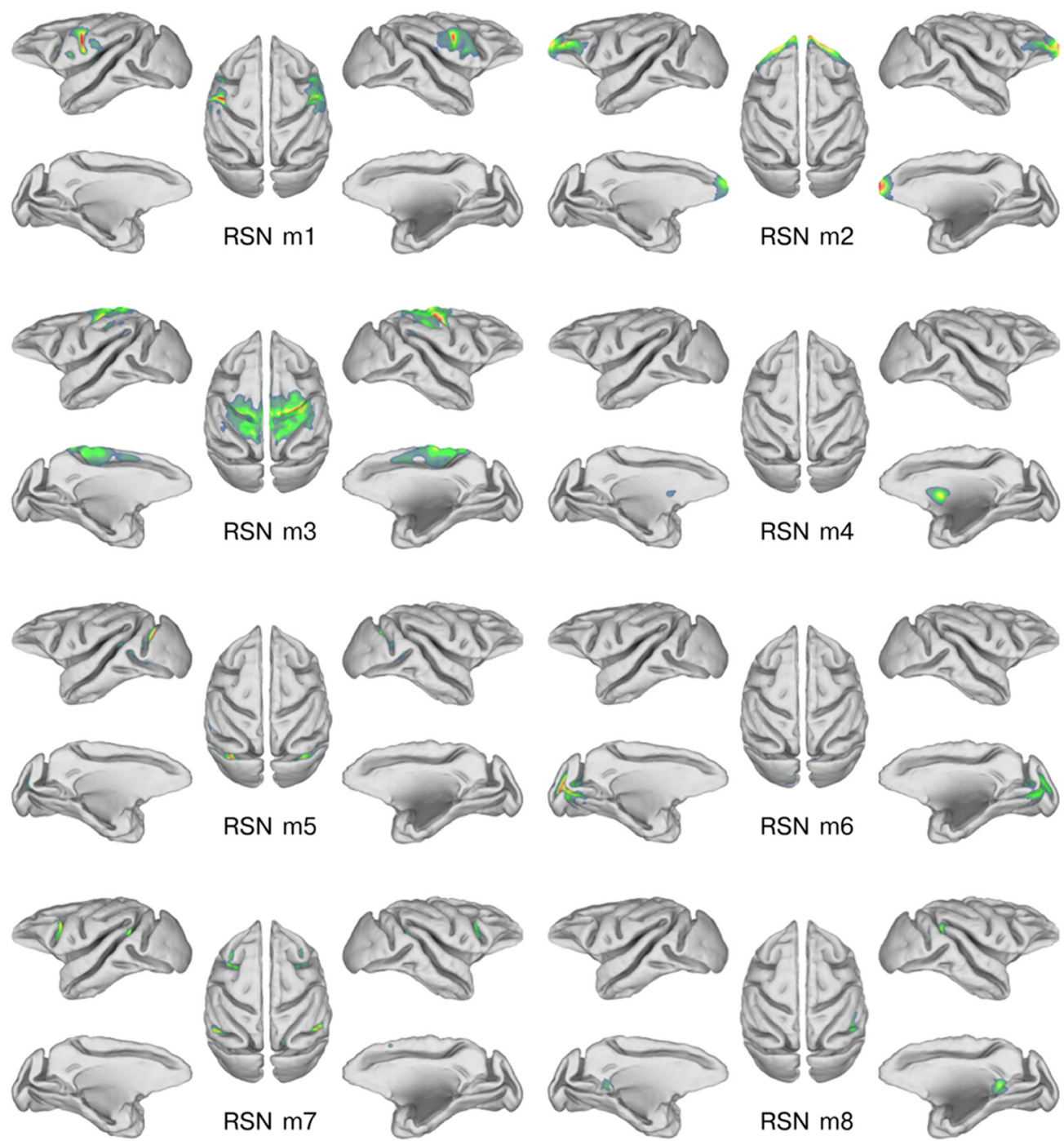

RSN m7
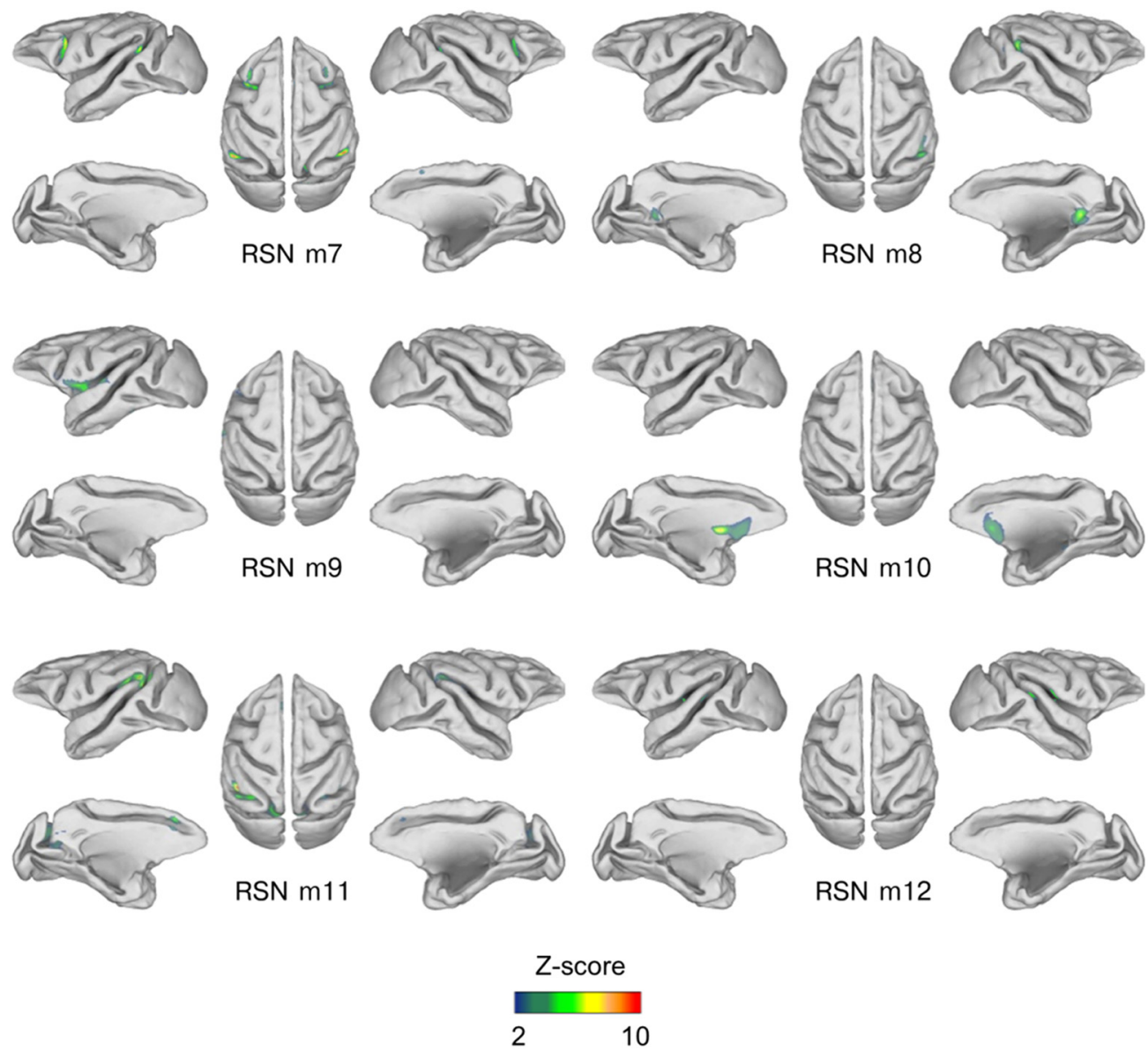

Figure 3. Folded cortical maps of the RSNs detected in monkeys. Each RSN is plotted (threshold at $Z>2$ ) over a representative cortical surface in dorsal, lateral, and medial views. 
Table 1. RSNs in monkeys and humans

\begin{tabular}{|c|c|c|c|c|}
\hline Code & Description & Consistency & Task-related fMRI study & Resting-state fMRI study \\
\hline \multicolumn{5}{|l|}{ Human RSNs } \\
\hline RSNh1 & Parafoveal visual & $24 / 24$ & (Tootell et al., 1998) & (Damoiseaux et al., 2006) \\
\hline RSNh2 & Ventral somatomotor & $24 / 24$ & (Moulton et al., 2009) & (Damoiseaux et al., 2006) \\
\hline RSN h4 & Dorsal somatomotor & $21 / 24$ & (Rao et al., 1995) & (Fox et al., 2006b) \\
\hline RSN h5 & Right frontoparietal & $21 / 24$ & (Koechlin et al., 1999) & (Beckmann et al., 2005) \\
\hline RSN h6 & Peripheral visual & $21 / 24$ & (Tootell et al., 1998) & (Damoiseaux et al., 2006) \\
\hline RSN h9 & Early auditory & $20 / 24$ & (Dhankhar et al., 1997) & (Mantini et al., 2007) \\
\hline RSN h10 & Ventral attention & $20 / 24$ & (Corbetta and Shulman, 2002) & (Fox et al., 2006a) \\
\hline RSN h11 & Left frontoparietal & $19 / 24$ & (Koechlin et al., 1999) & (Beckmann et al., 2005) \\
\hline RSNh12 & Default mode & $19 / 24$ & (Laird et al., 2009) & (Fox et al., 2005) \\
\hline RSN h13 & Lateral prefrontal & $18 / 24$ & (Braver et al., 1997) & (Beckmann et al., 2005) \\
\hline RSN h14 & Cingulo-insular & $18 / 24$ & (Dosenbach et al., 2006) & (Dosenbach et al., 2007) \\
\hline RSN m4 & - & $4 / 4$ & - & - \\
\hline RSN m5 & Peripheral visual & $4 / 4$ & (Brewer et al., 2002) & (Vincent et al., 2007) \\
\hline RSN m6 & Parafoveal visual & $4 / 4$ & (Brewer et al., 2002) & (Vincent et al., 2007) \\
\hline RSN m7 & Dorsal attention & $4 / 4$ & (Wardak et al., 2010) & (Vincent et al., 2007) \\
\hline RSN m8 & - & $3 / 4$ & - & - \\
\hline RSN m 9 & - & $3 / 4$ & - & - \\
\hline RSN m10 & - & $3 / 4$ & - & - \\
\hline RSN m11 & Default mode & $3 / 4$ & (Mantini et al., 2011) & (Hutchison et al., 2011) \\
\hline RSN m12 & Early auditory & $3 / 4$ & (Petkov et al., 2006) & (Hutchison et al., 2011) \\
\hline
\end{tabular}

We defined RSNs in the two species by means of ICA. The consistency of each RSN across subjects is indicated. We spatially compared the RSN maps with those published in previous resting-state and task-related fMRI. Whenever available, selected bibliographic references are also provided.

bore of the magnet. Human volunteers, in contrast, lay in a supine position during BOLD-fMRI scanning. MR-compatible headphones (Confon) with ear-cup pads (Joly et al., 2012) were used to protect subjects' ears from environmental noise, and to deliver the movie sound during natural vision. The subjects faced a translucent screen, onto which either a fixed red target with a diameter of 0.3 visual degrees (for resting state) or the movie "The Good, the Bad and the Ugly" in a window of $24 \times 10.2$ visual degrees (for natural vision) was rear projected from an LCD projector.

Data acquisition. For monkeys, fMRI was performed with a 3 T MR Siemens Trio scanner in Leuven, Belgium. The monkeys were scanned using an 8-channel phased array coil with SENSE reconstruction and a saddle-shaped, radial transmit-only surface coil. The functional images were collected using a gradient-echo $\mathrm{T} 2{ }^{*}$-weighted echo-planar sequence (40 slices, $84 \times 84$ in-plane matrix, TR/TE $=2000 / 19 \mathrm{~ms}$, flip angle $=$ $75^{\circ}$, voxel size $=1.25 \times 1.25 \times 1.25 \mathrm{~mm}^{3}$ ). For each monkey, 20 and 18 scanning runs of $10 \mathrm{~min}$ each were performed for resting state and natural vision, respectively. In addition, high-resolution, T1-weighted anatomical images were collected in separate sessions, to provide the anatomical reference for the functional scans. Under ketamine-xylazine anesthesia, an MP-RAGE sequence (TR/TE $=2200 / 4.06$, voxel size $=$ $0.5 \times 0.5 \times 0.5 \mathrm{~mm}^{3}$ ) was used to obtain nine whole-brain volumes, which were then averaged.

For human subjects, fMRI was performed with a 3 T MR Philips Achieva scanner in Chieti, Italy. The functional images were obtained using $\mathrm{T} 2{ }^{\star}$-weighted echo-planar images (EPI) with BOLD contrast using SENSE imaging. EPIs comprised 32 axial slices acquired in ascending order and covering the entire brain ( 32 slices, $230 \times 230$ in-plane matrix, $\mathrm{TR} / \mathrm{TE}=2000 / 35$, flip angle $=90^{\circ}$, voxel size $=2.875 \times 2.875 \times 3.5$ $\mathrm{mm}^{3}$ ). For each human subject, two and three scanning runs of $10 \mathrm{~min}$ ' duration were performed for resting state and natural vision, respectively. Furthermore, a 3D high-resolution T1-weighted image, to be used for anatomical reference, was collected by means of an MP-RAGE sequence $\left(\mathrm{TR} / \mathrm{TE}=8.1 / 3.7\right.$, voxel size $\left.=0.938 \times 0.938 \times 1 \mathrm{~mm}^{3}\right)$ at the end of the scanning session.
With both monkeys and humans, the eye position was monitored during fMRI scanning using a pupil-corneal reflection system at $120 \mathrm{~Hz}$ (Iscan). A calibration procedure was performed with the subject in the MR scanner, and before fMRI.

Eye data analysis. The eye position data (collected with a $120 \mathrm{~Hz}$ eyetracking system) were converted to visual degrees by a 4-point spatial projection calibration. For each resting-state run, monkeys were allowed to fixate within a window of $2 \times 3$ visual degrees. For each run, we defined the fixation rate as the ratio between the time in which the eye traces were inside the fixation window and the total duration of the run. We used a paired $t$ test to assess the difference in fixation rates between the two runs using human subjects. Next, we applied a one-way ANOVA to assess whether the fixation rate was different across monkeys. Finally, we used an unpaired $t$ test to assess differences between fixation rates in monkeys and humans. Results from monkey and human eye traces during the natural vision experiment have been reported in our previous study (Mantini et al., 2012b).

fMRI preprocessing. fMRI data preprocessing was performed with the SPM5.0 software package (http://www.fil.ion.ucl.ac.uk/spm), running under MATLAB (MathWorks). The functional image time series were preprocessed to compensate for slice-dependent time shifts, motion corrected within and across sessions, and linearly detrended, then coregistered to the anatomical image, and spatial normalized to atlas space. For the monkey data, the image template was the 112RM image (http://www.brainmap. wisc.edu/monkey.html) in monkey F99 atlas space (http://sumsdb.wustl. edu/sums/macaquemore.do). Conversely, theMNI152image(http://www. bic.mni.mcgill.ca/ServicesAtlases/HomePage) from the Montreal Neurological Institute (MNI) was used to define the human atlas space. The monkey and human atlas-transformed fMRI images were generated at spatial resolutions of 1 and $3 \mathrm{~mm}$ isotropic, respectively. Finally, they were spatially smoothed with a Gaussian kernel at 2 and $6 \mathrm{~mm}$ full-width-halfmaximum, respectively.

Resting-state network analysis. Independent RSN analyses were conducted on monkey and human fMRI datasets. Spatial ICA, a technique 


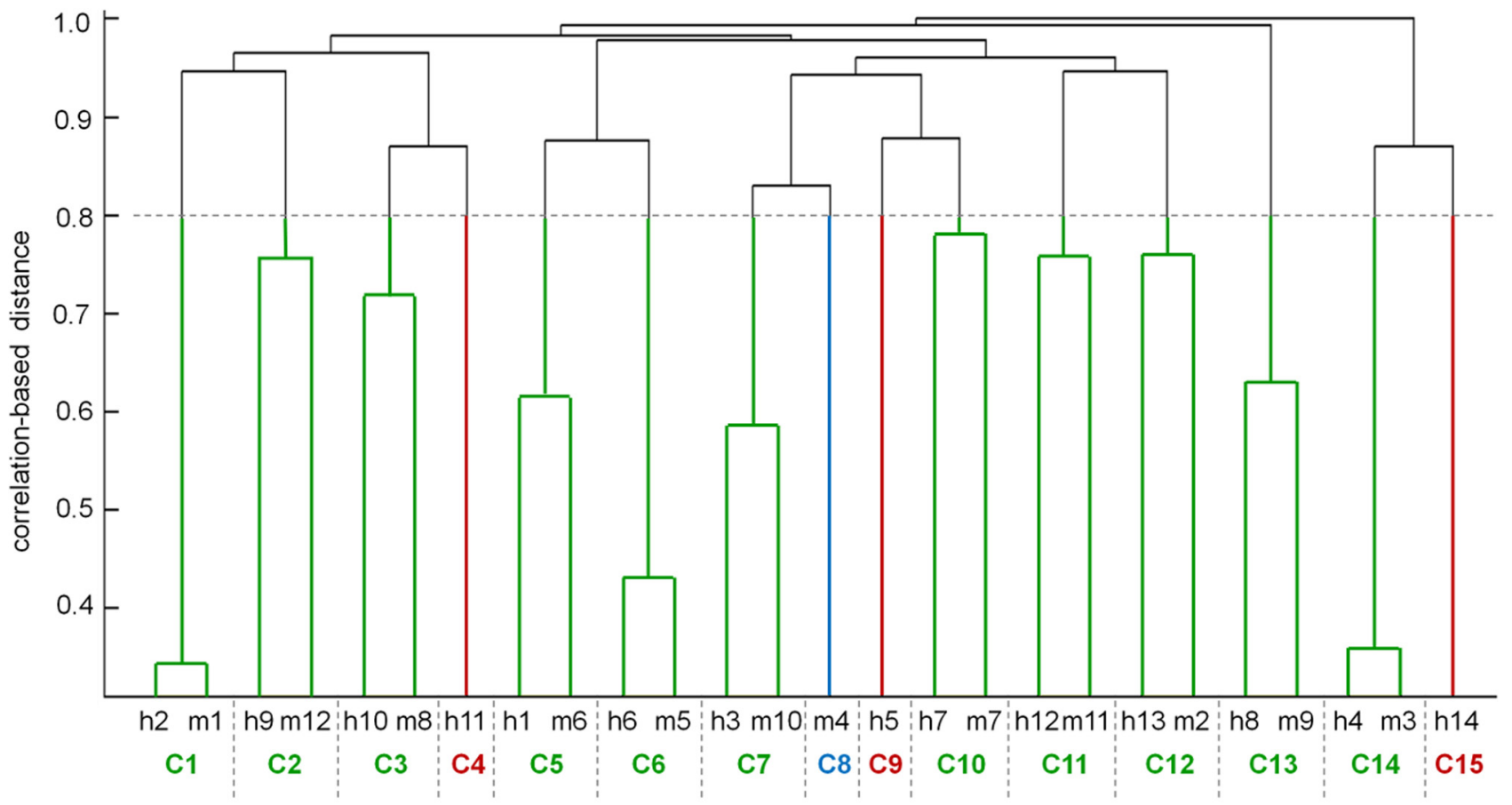

\begin{tabular}{|ll|}
\hline monkey-human clusters in sensory-motor regions & monkey-human clusters in associative regions \\
C1: ventral somatomotor & C3: ventral attention \\
C2: early auditory & C7: medial prefrontal \\
C5: parafoveal visual & C10: dorsal attention \\
C6: peripheral visual & C11: default-mode \\
C14: dorsal somatomotor & C12: lateral prefrontal \\
human-specific clusters & C13: language \\
C4: left fronto-parietal & \\
C9: right fronto-parietal & monkey-specific clusters \\
C15: cingulo--insular & C8: caudate/putamen \\
\hline
\end{tabular}

Figure 4. Spatial clustering of monkey and human RSNs. Among the 15 clusters detected, 11 contained RSNs of both species (spanning sensory-motor and associative regions). In addition, three clusters were composed of one human RSN and one cluster consisting of one monkey RSN.

Table 2. Robustness of the spatial clustering between monkey and human RSNs

\begin{tabular}{llllll}
\hline Cluster description & Human RSN & Monkey RSN & Intracluster correlation & Extracluster correlation & Clustering reliability \\
\hline Ventral somatomotor (C1) & RSN h2 & RSN m1 & $r=0.66$ & $r=0.07$ & $p<0.001$ \\
Dorsal somatomotor (C14) & RSN h4 & RSN m3 & $r=0.64$ & $r=0.05$ & $p<0.001$ \\
Parafoveal visual (C5) & RSN h1 & RSN m6 & $r=0.38$ & $r=0.07$ & $p<0.001$ \\
Peripheral visual (C6) & RSN h6 & RSN m5 & $r=0.52$ & $r=0.06$ & $p<0.001$ \\
Early auditory (C2) & RSN h9 & RSN m12 & $r=0.24$ & $r=0.09$ & $p=0.022$ \\
Ventral attention (C3) & RSN h10 & RSN m8 & $r=0.29$ & $r=0.09$ & $p=0.003$ \\
Medial prefrontal (C7) & RSN h3 & RSN m10 & $r=0.41$ & $r=0.07$ & $p<0.001$ \\
Dorsal attention (C10) & RSN h7 & RSN m7 & $r=0.22$ & $r=0.08$ & $p=0.032$ \\
Default mode (C11) & RSN h12 & RSN m11 & $r=0.24$ & $r=0.08$ & $p=0.022$ \\
Lateral prefrontal (C12) & RSN h13 & RSN m2 & $r=0.23$ & $r=0.07$ & $p=0.026$ \\
Language (C13) & RSN h8 & RSN m9 & $r=0.37$ & $r=0.07$ & - \\
Left frontoparietal (C4) & RSN h11 & - & - & $r=0.07$ & - \\
Right frontoparietal (C9) & RSN h5 & - & - & $r=0.07$ & - \\
Cingulo-insular (C15) & RSN h14 & - & - & $r=0.08$ & - \\
Putamen (C8) & - & RSN m4 & - & $r=0.06$ & -
\end{tabular}

For each cluster, we separately grouped the spatial correlations between the RSNs inside the cluster (intracluster correlations) and those between the RSNs of that cluster and all other RSNs (extracluster correlations). By averaging the correlations in these two groups, we defined representative intracluster and extracluster correlations, respectively (see Materials and Methods). Furthermore, we estimated the clustering reliability by means of a Mann-Whitney test between the correlations in the two groups. 


\section{monkey-human RSN clusters in sensory-motor regions}
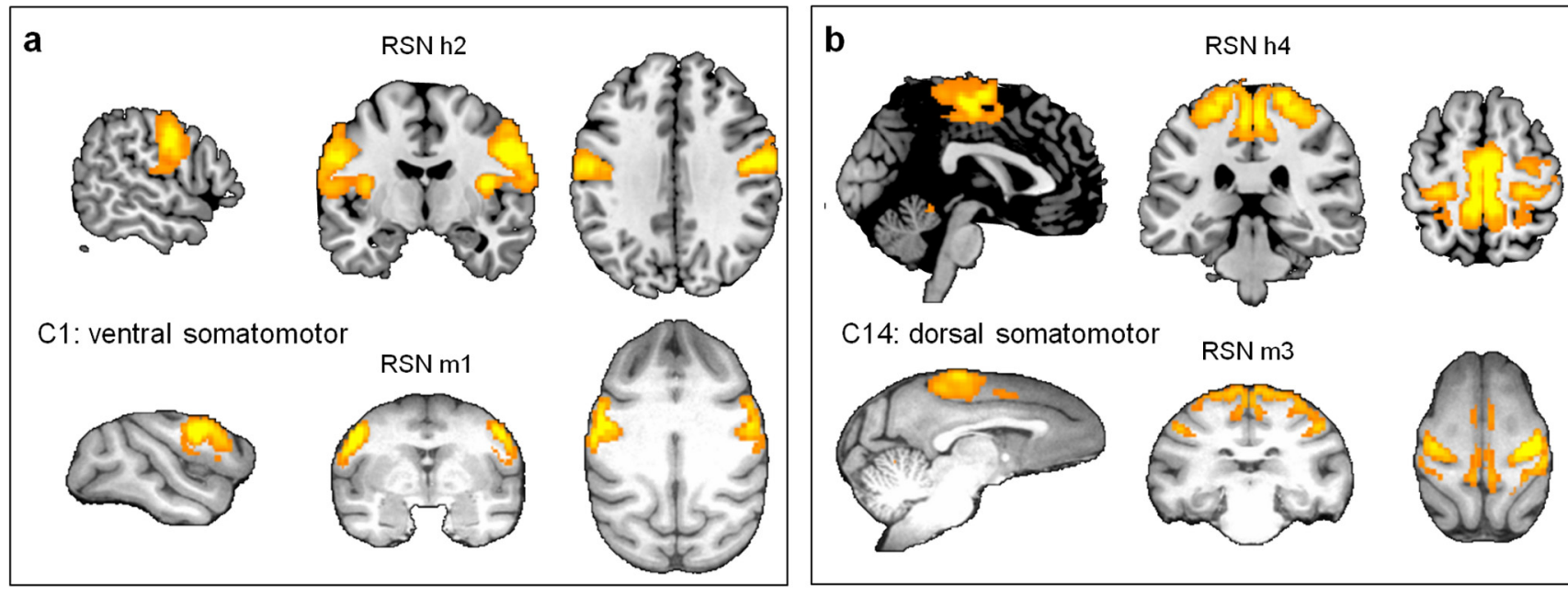

C14: dorsal somatomotor
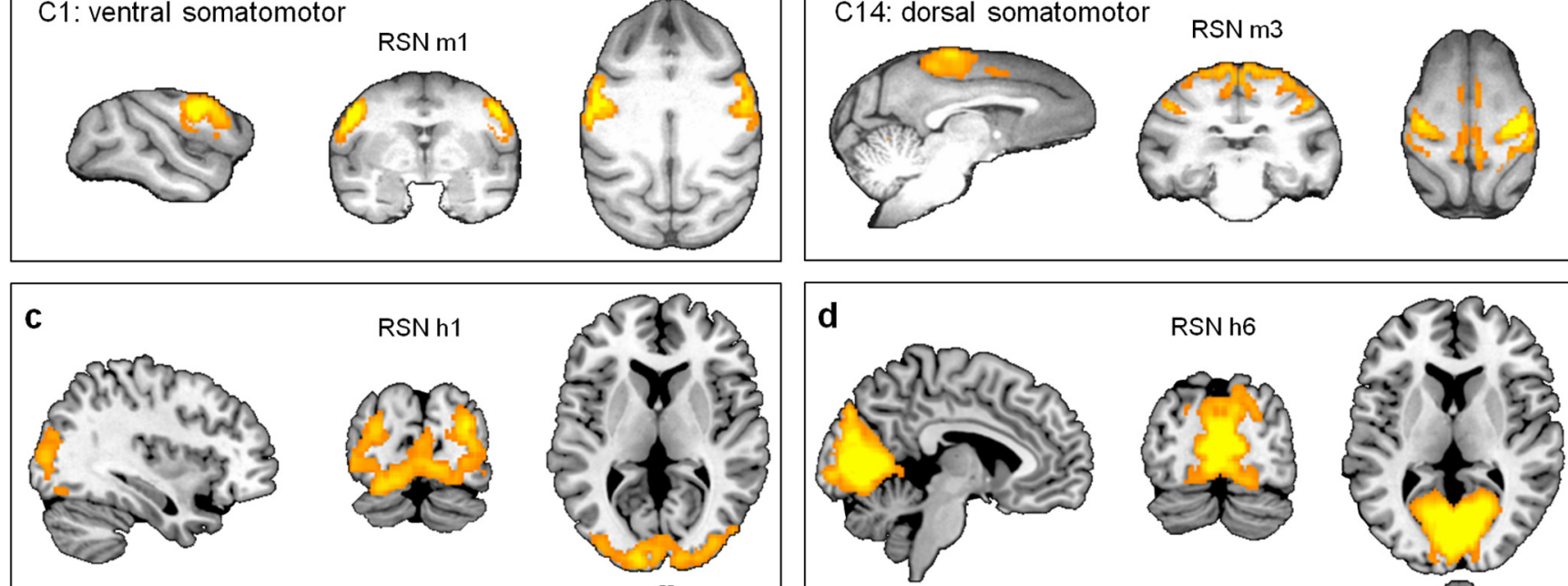

C5: parafoveal visual
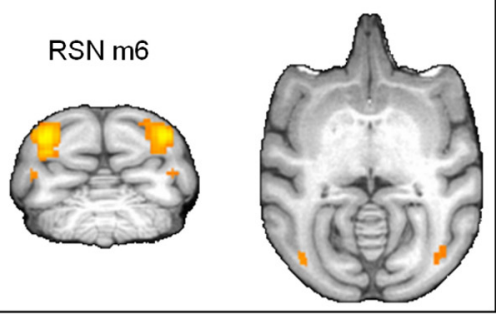

C6: peripheral visual
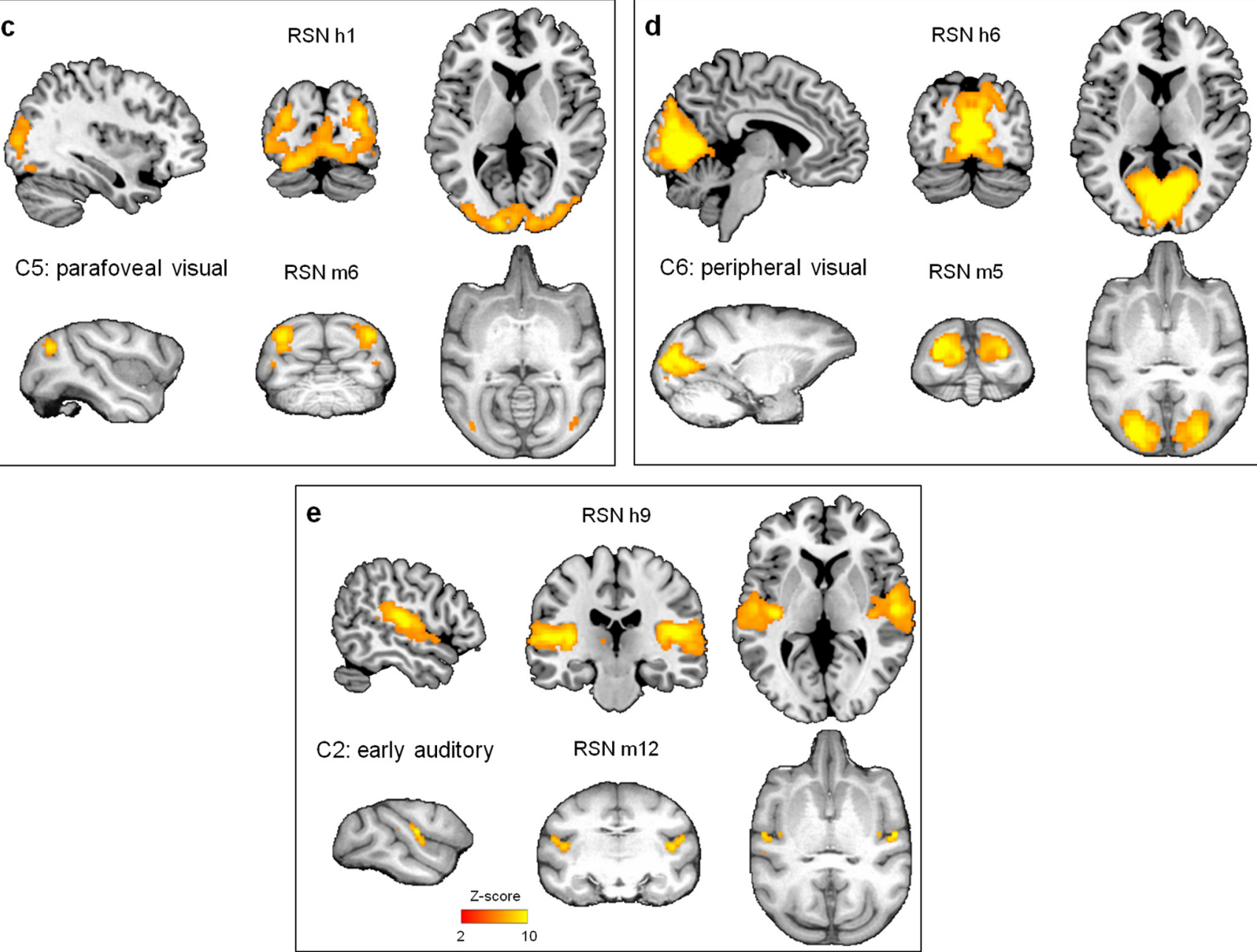

Figure 5. Clusters with monkey and human RSNs in sensory-motor regions. Among the 11 clusters containing RSNs of both species, 5 clusters encompassed sensory-motor regions. These were labeled as follows: (a) ventral somatomotor, (b) dorsal somatomotor, (c) parafoveal visual, (d) peripheral visual, and (e) early auditory. The maps of the constituent monkey and human RSNs (threshold at $Z>2$ ) are represented in sagittal, coronal, and axial views over an anatomical template of the respective species.

that extracts maximally independent patterns of coherent fMRI activity (Calhoun et al., 2001), was applied to each single dataset by means of the GIFT toolbox (http://icatb.sourceforge.net). The estimation of the number of independent components (ICs) was performed using the minimum description length criterion (Calhoun et al., 2001). After reduction of dimensionality by means of principal component analysis (accounting for at least $99.9 \%$ explained variance), ICs were retrieved by means of the FastICA algorithm, with a deflation approach and hyperbolic tangent (tanh) nonlinearity (Esposito et al., 2005). Each fMRI IC consisted of a waveform and a spatial map: the waveform corresponded to the time course of the specific pattern whereas the intensity of this activity across voxels was expressed by the associated spatial map. To display voxels 
contributing most strongly to a particular IC and to allow intersubject comparison, the intensity values in each map were scaled to $Z$-scores. To extend the ICA analysis from single to multiple datasets, the self-organizing group ICA (sogICA) method (Esposito et al., 2005) was used to sort the ICs extracted from different $\mathrm{fMRI}$ datasets and subsequently to average them to generate a single IC dataset. SogICA was applied according to a two-stage procedure: first to IC datasets from the same subject for the creation of a representative single-subject IC dataset (within-subject analysis), and then to single-subject IC datasets for the creation of a group-level IC dataset (acrosssubject analysis). For each sogICA procedure, the IC clusters with relative consistencies $\leq 50 \%$ or that were spatially correlated at $r>0.20$ with white matter or CSF patterns (as available in SPM5.0) were excluded from further analyses. The IC clusters obtained at the second level of sogICA were classified as resting-state networks. The RSN maps were represented in the volume space or in the surface space. The conversion from volumes to surfaces was performed with Caret 5.61 (http://brainvis.wustl.edu/ wiki/index.php/Caret:Download) (Van Essen and Dierker, 2007; Van Essen, 2012).

Topological correspondence between monkey and human networks. To determine spatial correspondences between the RSNs in monkeys and humans, we applied a monkey-to-human cortical deformation procedure to the monkey data based on anatomical landmarks (Van Essen and Dierker, 2007) and we performed hierarchical cluster analysis on the entire set of monkey and human RSNs in common space (Fig. 1). To characterize the clustering, we used the spatial correlation as a similarity metric and used the average linkage function. After the creation of the dendrogram, we selected the cutoff value for the graph yielding the maximum number of clusters with one monkey and one human RSN. This resulted in the definition of single- or two-element clusters. The former contained either a monkey or a human RSN with no correspondent detected in the other species; the latter contained RSNs putatively corresponding between species. To characterize the reliability of each cluster, we grouped the spatial correlations between the RSNs inside the cluster (intracluster correlations) and those between the RSNs of the cluster and all other RSNs (extracluster correlations). We statistically compared intracluster and extracluster correlations by means of the Mann-Whitney test, thus obtaining a quantitative measure of the cluster reliability.

Interspecies activity correlation between networks. The procedure for the detection of temporal correlations between fMRI responses in different species has been extensively described elsewhere (Mantini et al., 2012b). What follows is a summary of the analysis steps.

We convolved the monkey and human fMRI time courses with a canonical human or monkey hemodynamic response function, respectively, to allow for different hemodynamic peak delays and spectral contents. We calculated a representative time course for each RSN in each subject by averaging the signals across the voxels of the network map (threshold at $Z>2$ ). To estimate the relative contribution of stimulusdriven activity, we calculated temporal correlations of RSN time courses across subjects, or intersubject correlation within a species (Hasson et al., 2004; Mantini et al., 2012b). To calculate their statistical significance while controlling for signal autocorrelation, we used a nonparametric approach based on permutation testing with 10,000 iterations, based on surrogate time courses with a similar spectrum and value distribution (Schreiber and Schmitz, 1996). Subsequently, we selected the monkey and human RSNs with significant stimulus-driven activity and con- monkey-human RSN clusters in sensory-motor regions

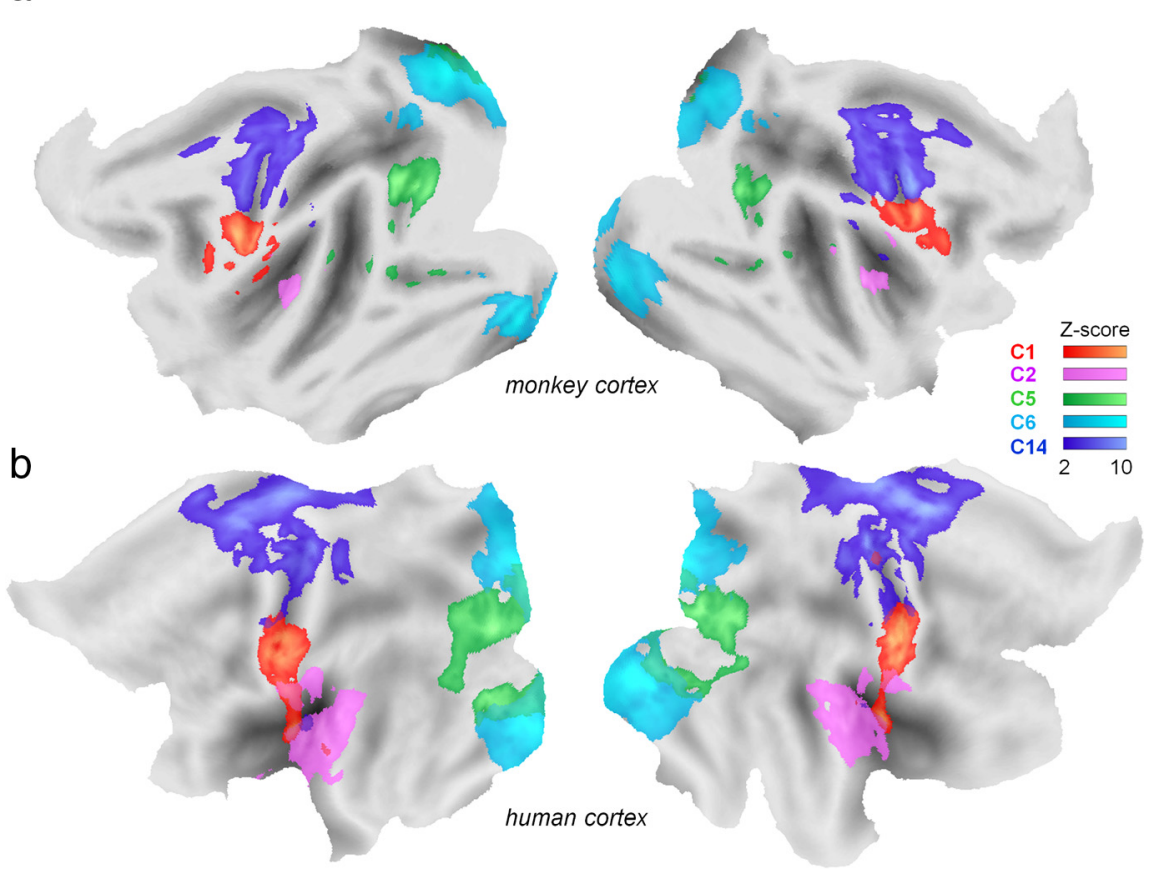

Figure 6. Topologically corresponding monkey and human RSNs in sensory-motor regions. The constituent (a) monkey and (b) human RSNs belonging to the five clusters encompassing sensory-motor regions are represented with different colors over flattened cortical surfaces of their respective species (threshold at $Z>2$ ).

structed a unique RSN time course for each of them by averaging the corresponding single-subject time courses. This allowed us to maximize the relative contribution of the stimulus-evoked responses above spontaneous activity. Next, we examined the similarity of RSN time courses within each species by means of hierarchical clustering (Bartels and Zeki, 2005). This allowed us to directly compare hierarchical similarities across species on a functional basis. By using the RSN time courses, we also performed an interspecies activity correlation (ISAC) analyses. For each human RSN, we calculated correlations among non-conspecific voxels to reveal regions displaying similar functional processing activity in a spatially unconstrained manner (Mantini et al., 2012b). Furthermore, we computed a matrix of partial correlations between monkey and human RSN time courses to delineate similarities in the stimulus-related responses. Again, we estimated the statistical significances of interspecies correlations by permutation testing, following the same nonparametrical approach used for intersubject correlations. We did not apply corrections for multiple comparisons on the probabilities associated with partial correlations; however, our partial-correlation approach minimized the detection of false significant correlations arising from indirect temporal similarities with other networks. To verify the robustness of our results, we divided the movie fMRI data into three parts, each corresponding to a movie clip. For each individual part, we repeated the statistical analysis described above, and then assessed the consistency of significant correlations across movie clips.

Topological and functional correspondence between monkey and human networks. After independently assessing putative topological and functional correspondences across monkey and human networks, we examined whether any relationship existed between the two. We calculated all pairwise spatial correlations between each human RSN and the monkey RSNs, then selected the highest correlation as an index of topographical correspondence. We used exactly the same procedure to assess the functional correspondence using the ISAC correlations between each human RSN and all monkey RSNs. This approach allowed us to characterize in better detail the human networks showing high topological and functional correspondences (supposedly conserved across evolution) from other cases in which relatively low 
monkey-human RSN clusters in associative regions
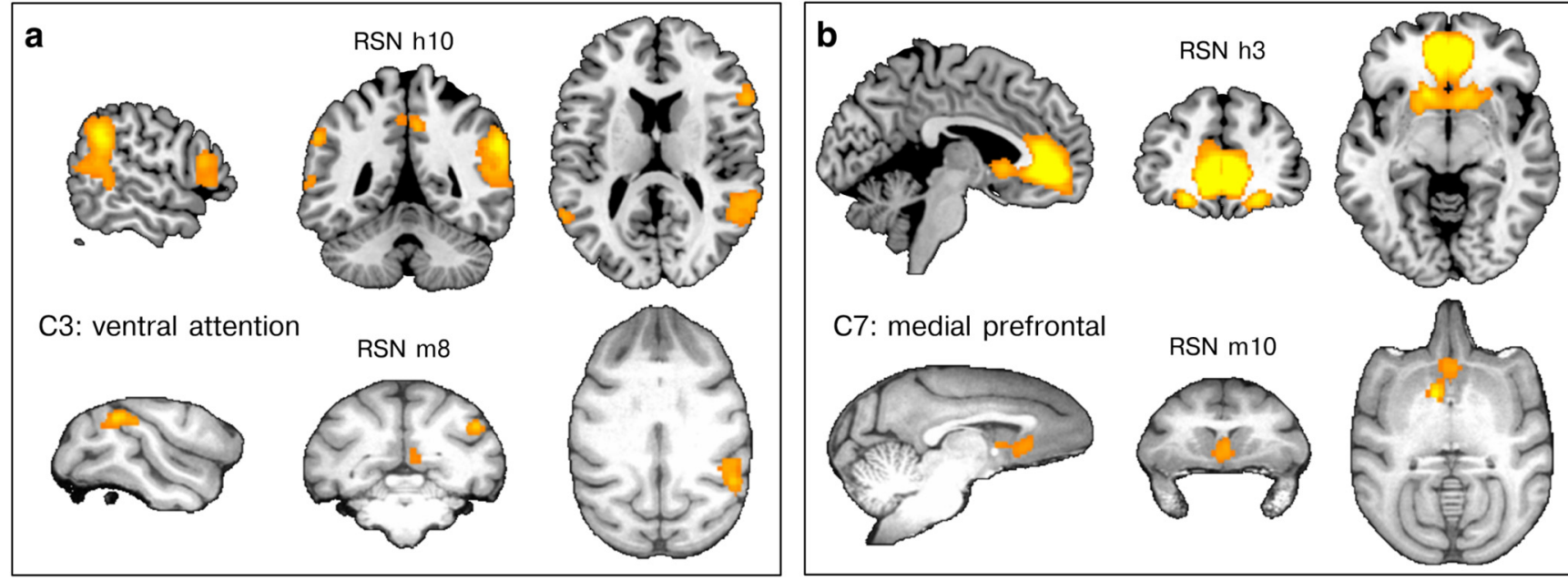

C7: medial prefrontal
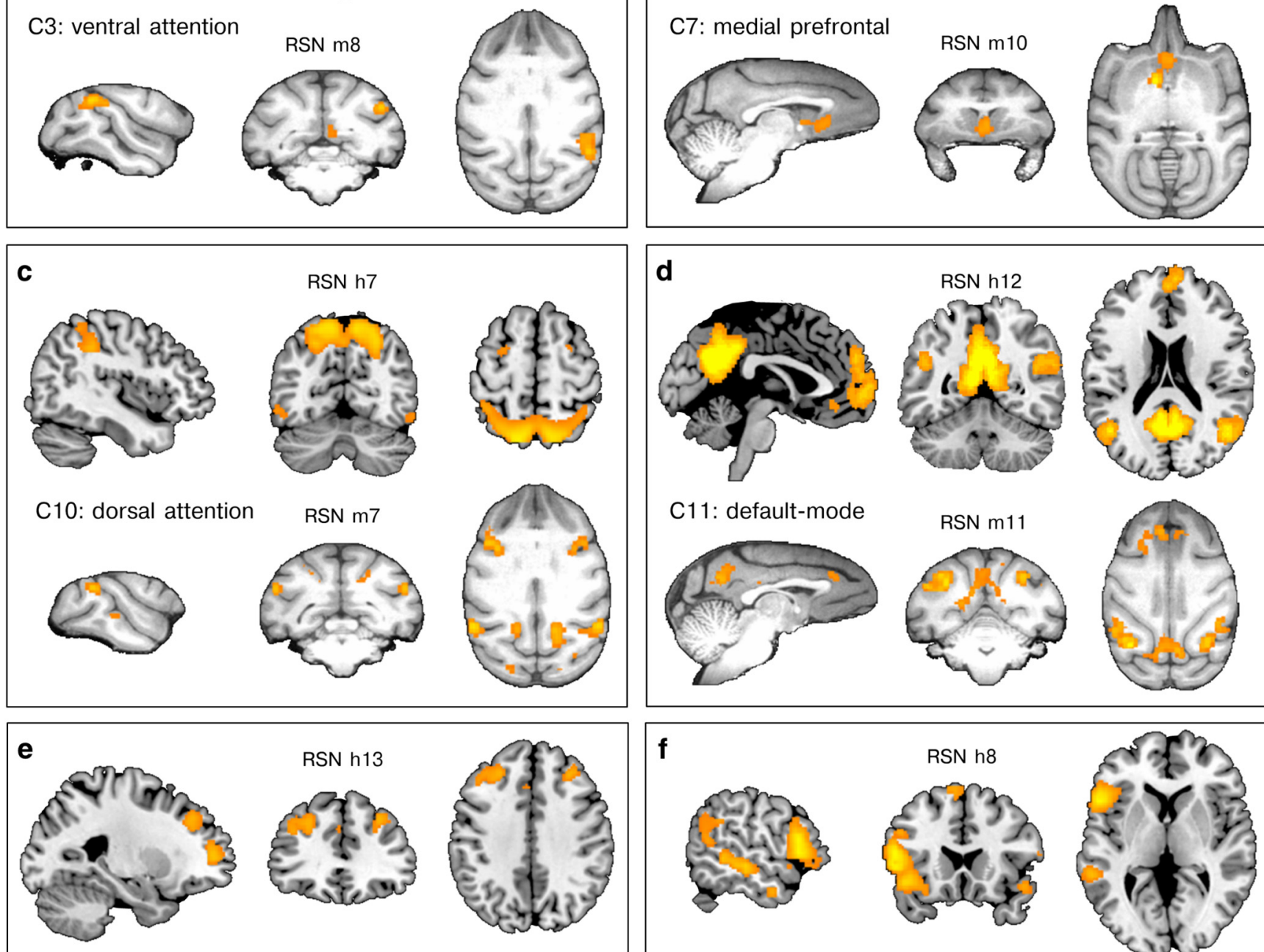

\section{C12: lateral prefrontal}
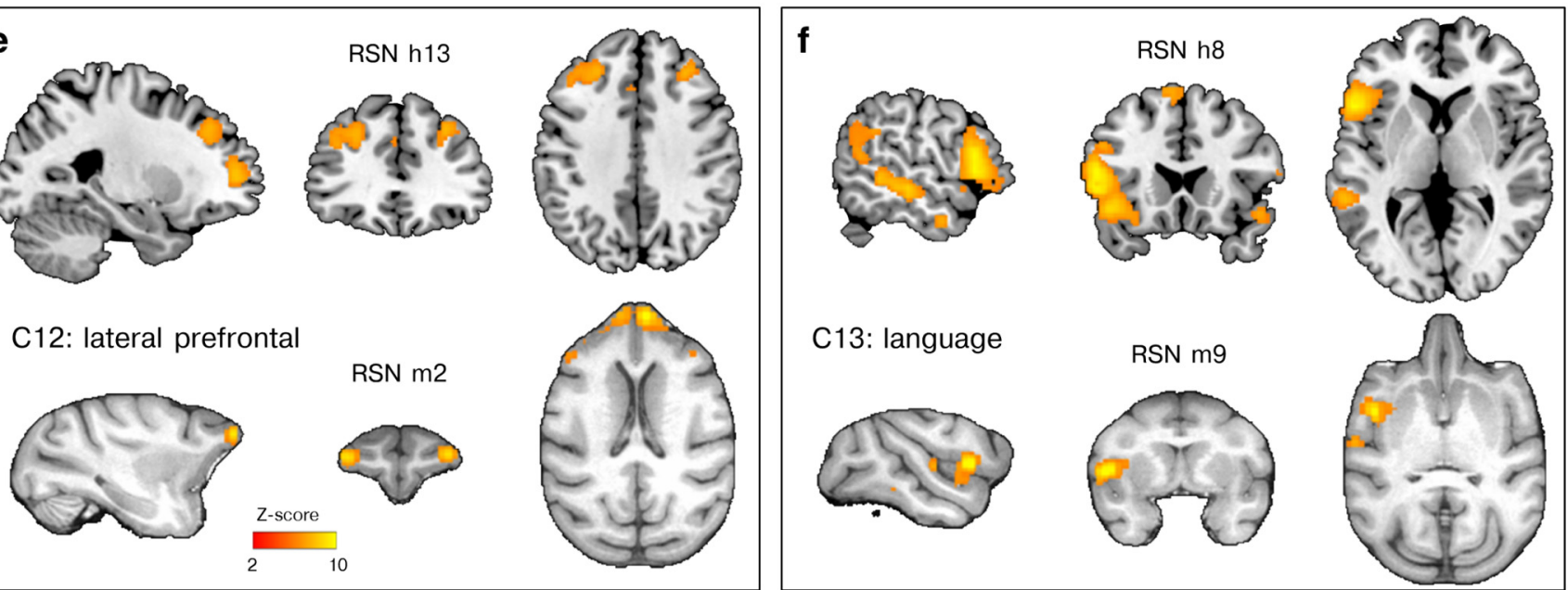

Figure 7. Clusters with monkey and human RSNs in associative regions. Among the 11 clusters containing RSNs of both species, 6 clusters encompassed associative regions. These were labeled as follows: (a) ventral attention, (b) medial prefrontal, (c) dorsal attention, (d) default-mode, (e) lateral prefrontal, and ( $\boldsymbol{f}$ ) language. The maps of the constituent monkey and human RSNs (threshold at $Z>2$ ) are represented in sagittal, coronal, and axial views over an anatomical template of the respective species.

topological correspondence or functional correspondence had been observed. At a more speculative level, human networks with little interspecies correspondence with respect to both domains might be evolutionarily novel, whereas networks with high topological but low functional correspondence might indicate functional redeployment.

\section{Results}

RSNs in macaques and humans

During resting-state scanning, the fixation rate averaged 93.3 and $92.6 \%$ in human and nonhuman subjects, respectively. The 
human-subject fixation rate was similar between runs (paired $t$ test, $t_{(46)}=1.3, p=$ $0.199)$. The four monkeys showed a more variable, but still high, fixation rate within runs, ranging between 90.6 and $96.3 \%$ (one-way ANOVA, $F_{(3,76)}=5.769, p=$ 0.001). Furthermore, we detected no significant differences in fixation rates between the groups of monkeys and humans (unpaired $t$ test, $t_{(126)}=0.831, p=0.407$ ). By using ICA on the resting-state fMRI data, we identified 12 monkey and 14 human RSNs. With the exception of one subcortical monkey network, all RSNs comprised distinct regions of the cortex, in both monkeys and humans (Figs. 2, 3). A limited portion of the cortex, including inferior temporolateral and ventrolateral prefrontal regions, was only partially included within the RSNs. The RSNs were for the most part consistent, and were present in at least $75 \%$ of the subjects (see Table 1 for details). We tentatively labeled these networks using information from previous studies (Table 1). We observed that the RSNs in humans mostly corresponded to those reported in the literature. In some cases, our ICA method yielded a finer parcellation of cortical networks than reported in previous studies. For example, we retrieved two visual networks, one parafoveal and one peripheral (Damoiseaux et al., 2006), that are typically revealed by eccentricity maps in retinotopic mapping studies (Kolster et al., 2009, 2010), while a single visual network in the occipital cortex is often reported in resting-state studies (Lowe et al., 1998; Mantini et al., 2007; van den Heuvel et al., 2009). In contrast to human RSNs, very limited knowledge concerning the topology of RSNs in awake, resting macaques is currently available (Moeller et al., 2009). Overall, however, our RSNs showed a high degree of spatial correspondence with monkey networks in the anesthetized state (Vincent et al., 2007; Hutchison et al., 2011), suggesting that network topology reflects, at least to some extent, the underlying structural architecture of the brain (Skudlarski et al., 2008).

\section{Topological correspondence between monkey and human networks}

We performed a spatial clustering analysis of RSN maps (Fig. 1), to define clusters of activity belonging to corresponding RSNs in the two species, as well as clusters belonging to a monkey or human RSN, with no counterpart in the other species. Accordingly, the criterion for belonging to a cluster did not allow two or more human RSNs to correspond to a single monkey RSN, or vice versa. The spatial clustering of monkey and human RSNs resulted in the definition of 15 clusters in total (Fig. 4). Of these, 11 contained spatially corresponding RSNs in the two species (Table 2). Five of these 11 clusters comprised early auditory, visual, and somatomotor regions, and showed striking topological interspecies similarities (Figs. 5, 6). The visual systems of both humans and monkeys were divided into two RSNs corresponding to the parafoveal and the more peripheral visual field representations (Orban et al., 2004; Fig. 6). For both species, the somatomotor networks were also partitioned into dorsal and ventral subdivisions (Graziano, 2006; Fig. 6). Overall, the clusters including monkey-human RSN clusters in associative regions

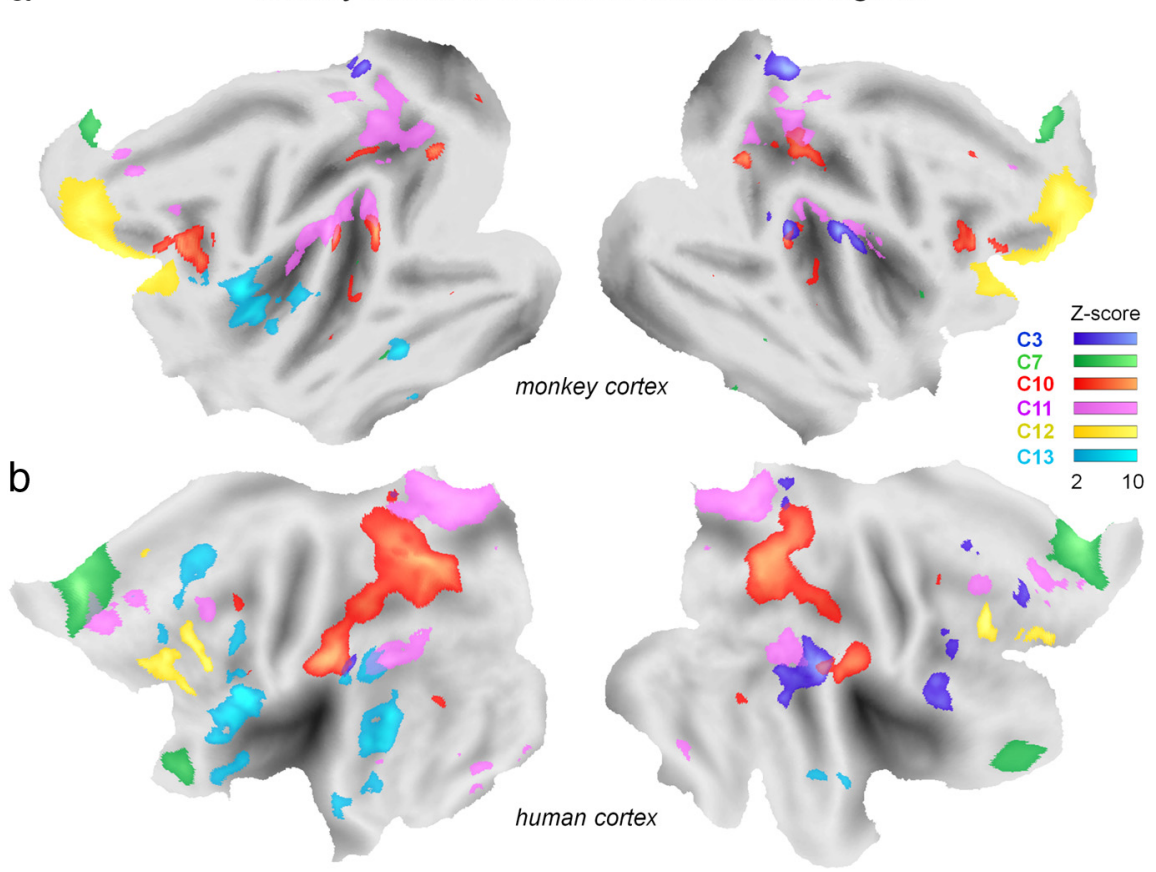

10

Figure 8. Topologically corresponding monkey and human RSNs in associative regions. The constituent (a) monkey and (b) human RSNs belonging to the six clusters comprising associative regions are represented with different colors over the flattened cortical surfaces of their respective species (threshold at $Z>2$ ).

these RSNs were highly consistent and characterized by high intracluster correlations (Table 2).

The remaining six clusters spanned higher-order sensory and associative cortical regions (Figs. 7, 8). Among these RSNs, the default-mode (Vincent et al., 2007; Laird et al., 2009; Mantini et al., 2011) and the dorsal attention network (Corbetta and Shulman, 2002; Vincent et al., 2007) have already been described in both species (Fig. $7 c, d$ ). Two other monkey-human clusters included topologically corresponding medial and lateral prefrontal networks (Fig. 7b,e), respectively, consistent with earlier proposed functional-anatomic subdivisions of the primate frontal cortex (Rushworth et al., 2011). On the other hand, we found other human RSNs clustered with monkey RSNs for which little or no information is available. The spatial clustering approach revealed particularly well the monkey correspondents for human ventral attention (Corbetta and Shulman, 2002) and language networks (Price, 2000; Fig. 7a,f). Interestingly, not all the constituent areas of the human RSNs were present in the monkey RSN belonging to the same cluster. For example, we observed the ventrolateral prefrontal cortex in the human language network and in its putative monkey correspondent, whereas temporal areas were much more closely integrated into the human language network (Figs. $7 f, 8$ ). Also, while the human ventral attention network included mainly the temporoparietal junction and the inferior frontal gyrus in the right hemisphere, its putative monkey correspondent included only the right temporoparietal cortex, but no frontal region (Figs. 7a, 8).

Our cluster analysis also produced one monkey-specific and three human-specific clusters (Fig. 9). The monkey-specific cluster was composed of a monkey subcortical network, mainly including caudate and putamen. The human networks with no topological counterparts in the monkey were located in cortical regions having larger than average cortical expansions (Striedter, 2002; Van Essen and Dierker, 2007; Fig. 10). Of these, two were 
clusters containing one human RSN
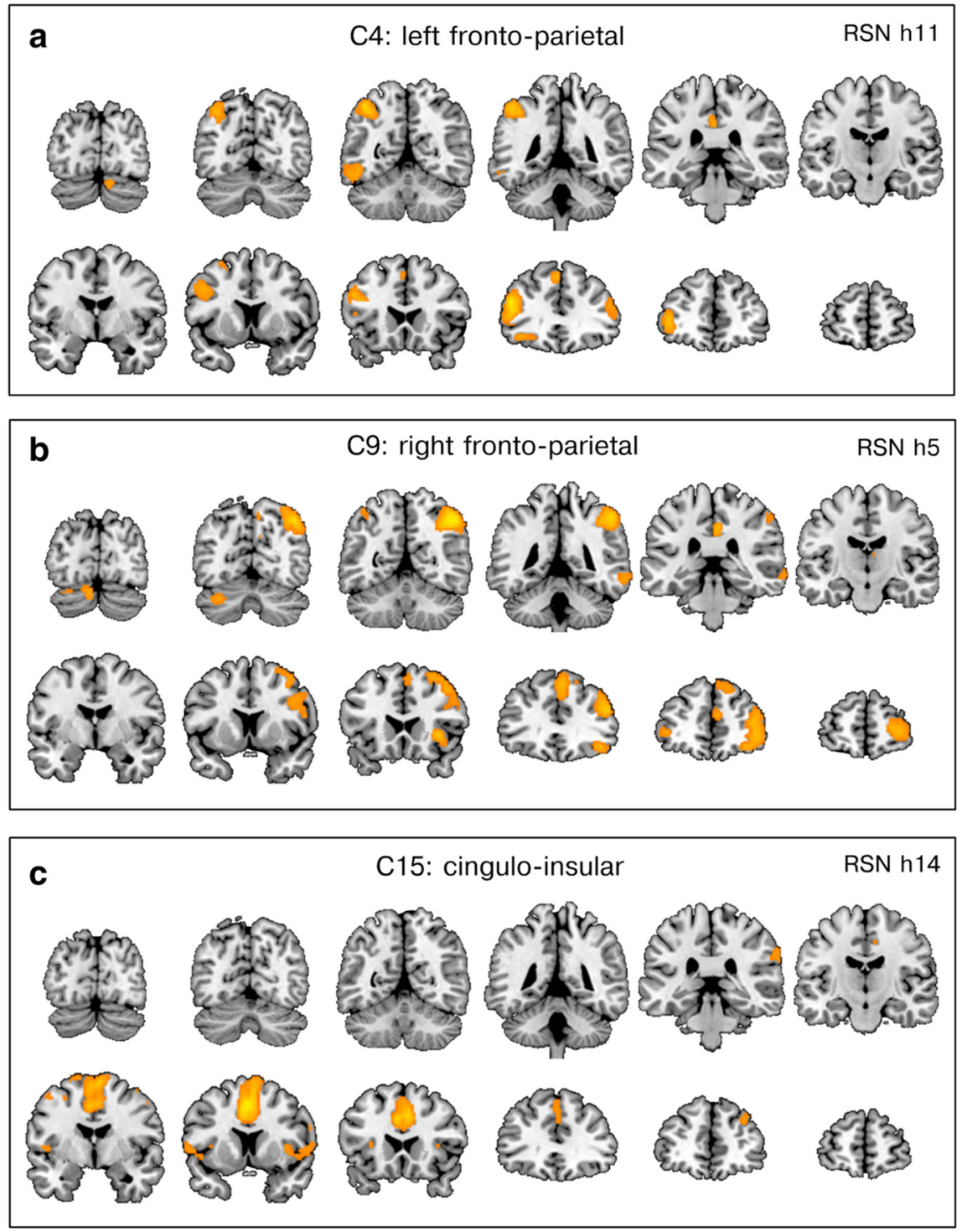

clusters containing one monkey RSN

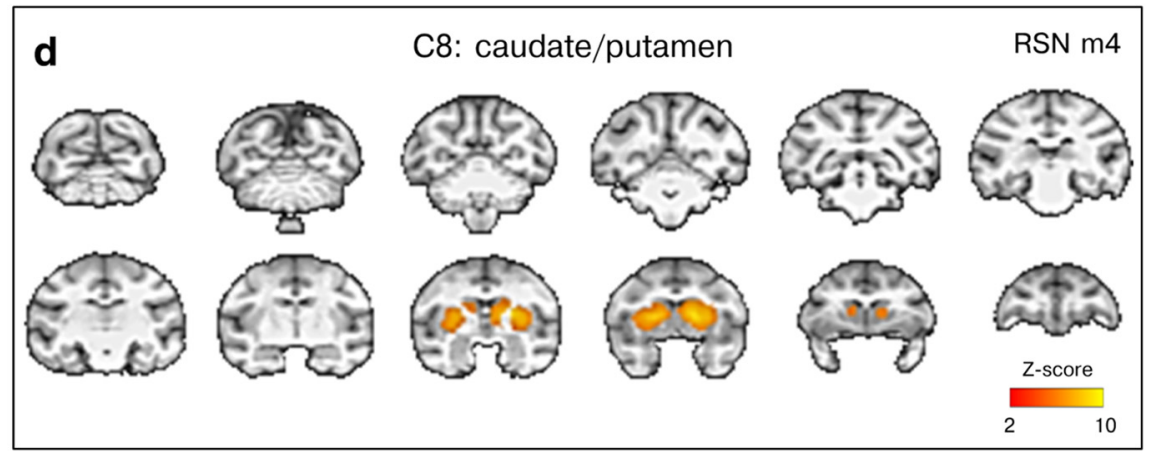

Figure 9. Clusters containing a single human or a single monkey RSN. The three clusters that contained only one human RSN were labeled as follows: ( $\boldsymbol{a}$ ) left frontoparietal, $(\boldsymbol{b})$ right frontoparietal, and (c) cingulo-insular. $\boldsymbol{d}$, An additional single-element cluster contained a subcortical monkey RSN, consisting mainly of caudate and putamen. The maps of the monkey and human RSNs (thresholded at $Z>2$ ) are represented in coronal views over an anatomical template of the respective species.

largely symmetrical to one another and encompassed portions of prefrontal and parietal cortex, such as the middle frontal gyrus, the inferior operculum, and the horizontal segment of the intraparietal sulcus (Figs. 9a,b,10a). The third human-specific net- work was bilateral, and mainly included the anterior insula and the dorsal anterior cingulate (Figs. $9 c, 10 a$ ).

Functional correspondence between monkey and human networks After identifying the networks that are topologically correspondent between species, we ascertained the degree of similarity in their functional responses. We also examined whether networks found only in the human might have functionally similar counterparts, but located at different cortical sites in the monkey. First, we calculated intersubject correlations (Hasson et al., 2004; Mantini et al., 2012b) to test for the presence of consistent stimulus-evoked fMRI responses $(p<0.001)$. Although the comprehension of the movie is not comparable between monkeys and humans (Passingham, 2009; Shepherd et al., 2010), this analysis showed that all 12 monkey and 14 human networks were effectively driven by the stimuli (see Table 3 for details). Following the ISAC approach (Mantini et al., 2012b), we corrected the monkey and human network time courses for the difference in the hemodynamic response, and compared the resulting signals between species.

We first examined similarities in RSN time courses of monkeys and of humans, as measured by canonical correlations. We conducted a hierarchical clustering analysis separately on the human and monkey data, which revealed patterns of functional interactions between networks during natural vision and provided a means to qualitatively and indirectly compare cortical networks in the two species. The resulting dendrograms showed a number of converging features between monkeys and humans (Fig. 11). For instance, parafoveal and peripheral visual networks displayed similar time courses, as well as dorsal and ventral attention networks, and dorsal and ventral somatomotor networks. Also, the default-mode network was coupled with frontal networks in both monkeys and humans. On the other hand, the hierarchical clustering analysis suggested that the processing of the stimuli may not be entirely identical between species. For example, the visual networks were functionally related to the attention networks more in monkeys than in humans. Also, the human language and ventral somatomotor networks showed largely dissimilar time courses, whereas their topographically correspondent networks in the monkey were quite strongly coupled. These results suggested that some cortical networks may 
be functionally similar between monkeys and humans, while others may not.

To directly test for this possibility, we used the ISAC approach and quantified functional correspondences between cortical networks in the two species. Unlike our previous ISAC studies (Mantini et al., 2012a,b), we calculated partial correlations, rather than canonical correlations, to control for the effects of interactions across networks in the same species (Fig. 11). For illustrative purposes, we have extracted the time courses from three selected networks in both species (foveal visual, peripheral visual, and early auditory) and we have directly compared the matrices containing canonical and partial correlations, respectively (Fig. 12). We used permutation testing (see Materials and Methods) to evaluate the significance of the partial correlations $(p<0.05)$, to determine whether human networks had reliable functional correspondents in the monkey. This functional analysis revealed that the parafoveal and the peripheral visual, the early auditory, the ventral somatomotor, and the ventral attention networks exhibited consistent temporal correlation with their topological correspondent in the monkey (Fig. 13). The response of the human auditory network also had slight temporal similarity with that of the monkey language network, which reached significance $(p<0.05)$ in only one out of three movie blocks. Among the other human networks having a topological correspondent in the monkey, the dorsal somatomotor, the language, the medial prefrontal, and the lateral prefrontal networks showed no similarity in the interspecies functional responses, whereas the responses of the dorsal attention and the default mode networks were significantly correlated between species $(p<0.05)$ for only one movie clip. Finally, the two human-specific frontoparietal networks showed functional signatures completely distinct from any monkey network, whereas the human cingulo-insular network was significantly correlated with the ventral somatomotor network in macaques in one out of the three movie clips (Fig. 13). Theoretically, it could be possible that functional equivalents of the two human frontoparietal and the cinguloinsular networks exist in the monkey, but that they were missed by our ISAC analysis because the latter was restricted to the cortical territory covered by the ICA-based RSNs. To test this possibility, we performed a seed-based ISAC analysis, correlating the average time courses in the human-specific RSNs across non-conspecific voxels. This analysis revealed no voxels in the monkey showing significant correlation $(q<0.05)$ with any of the human frontoparietal and the cingulo-insular networks, further supporting the concept that these networks are human specific.

\section{Assessing topological and functional correspondences between species}

The distinction between human networks with and without monkey correspondents should not be taken as absolute, be-
Table 3. Analysis of network responses across subjects

\begin{tabular}{lllll}
\hline Cluster description & $\begin{array}{l}\text { Human } \\
\text { RSN }\end{array}$ & $\begin{array}{l}\text { Inter-subject } \\
\text { correlation }\end{array}$ & $\begin{array}{l}\text { Monkey } \\
\text { RSN }\end{array}$ & $\begin{array}{l}\text { Intersubject } \\
\text { correlation }\end{array}$ \\
\hline Ventral somatomotor (C1) & RSN h2 & $r=0.27$ & RSN m1 & $r=0.22$ \\
Dorsal somatomotor (C14) & RSN h4 & $r=0.27$ & RSN m3 & $r=0.17$ \\
Parafoveal visual (C5) & RSN h1 & $r=0.52$ & RSN m6 & $r=0.58$ \\
Peripheral visual (C6) & RSN h6 & $r=0.52$ & RSN m5 & $r=0.64$ \\
Early auditory (C2) & RSN h9 & $r=0.62$ & RSN m12 & $r=0.22$ \\
Ventral attention (C3) & RSN h10 & $r=0.38$ & RSN m8 & $r=0.22$ \\
Medial prefrontal (C7) & RSN h3 & $r=0.21$ & RSN m10 & $r=0.15$ \\
Dorsal attention (C10) & RSN h7 & $r=0.40$ & RSN m7 & $r=0.64$ \\
Default mode (C11) & RSN h12 & $r=0.31$ & RSN m11 & $r=0.44$ \\
Lateral prefrontal (C12) & RSN h13 & $r=0.19$ & RSN m2 & $r=0.31$ \\
Language (C13) & RSN h8 & $r=0.44$ & RSN m9 & $r=0.20$ \\
Left frontoparietal (C4) & RSN h11 & $r=0.26$ & - & - \\
Right frontoparietal (C9) & RSN h5 & $r=0.28$ & - & - \\
Cingulo-insular (C15) & RSN h14 & $r=0.22$ & - & - \\
Putamen (C8) & - & - & RSN m4 & $r=0.16$ \\
\hline
\end{tabular}

All networks showed significant intersubject correlations across subjects (permutation testing, $p<0.001$ ), suggesting the presence of substantial stimulus-related activity.

cause interspecies correspondences are measured by correlations on a continuous scale. We therefore quantified the degree of correspondence, based on a combination of spatial and temporal measures, of each human network with all monkey networks (Fig. 14). Networks located in the upper right corner in this figure were topographically and functionally very similar between species, whereas those in the lower left corner were more dissimilar. We observed that the visual networks and the ventral somatomotor network were topologically and functionally similar between species; conversely, similarities in the attention networks and the auditory networks were less marked. The human dorsal somatomotor net- 
a

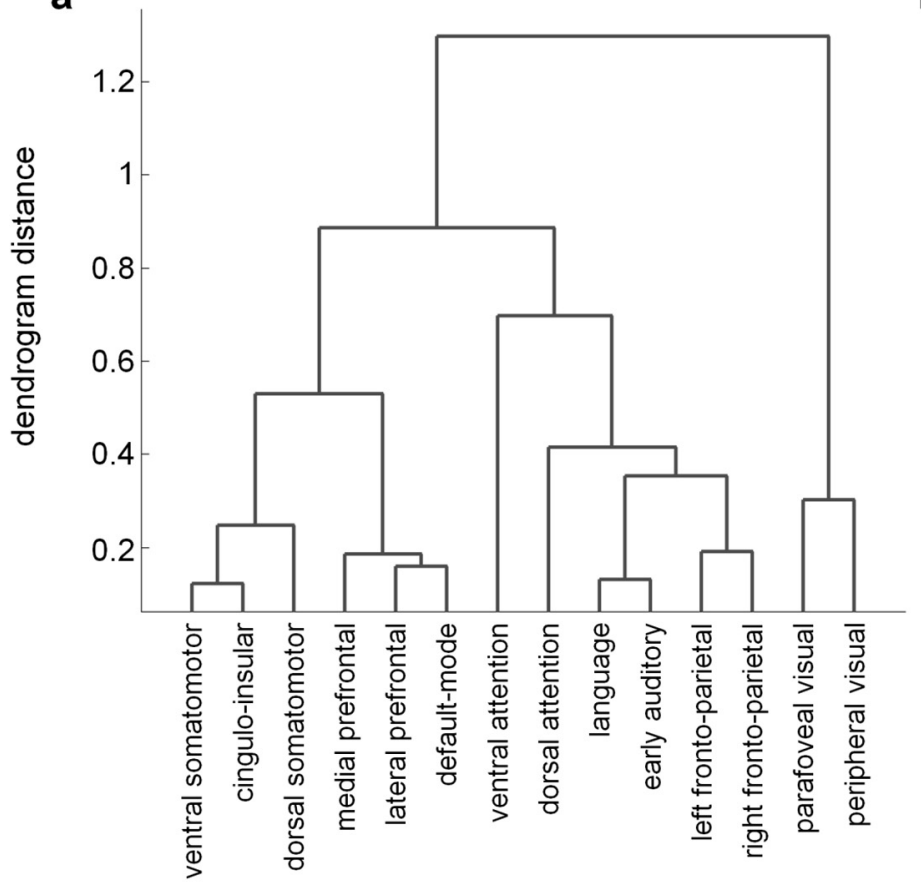

b

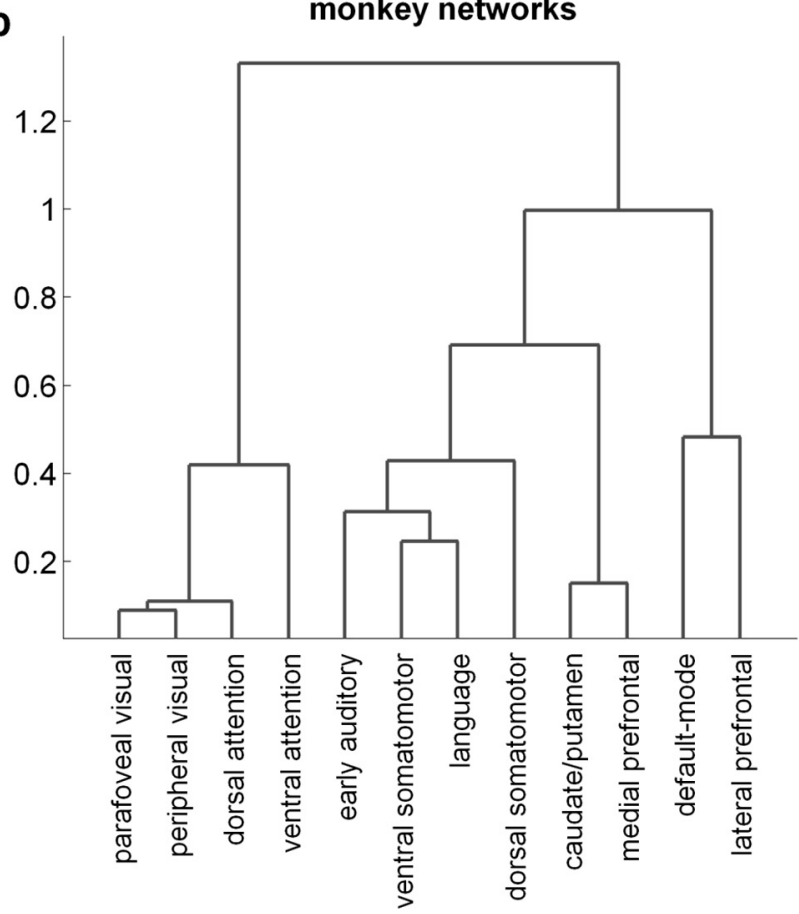

Figure 11. Hierarchical functional relationships between cortical networks of monkeys and humans during natural vision. A clustering algorithm was used to group the time courses of the RSNs in each of the two species, using the temporal correlation as similarity metric. $\boldsymbol{a}$, Dendrogram representing functional relationships between humans RSNs. $\boldsymbol{b}$, Dendrogram representing functional relationships between monkey RSNs.

work showed high spatial correlation with a monkey network encompassing topologically corresponding regions; yet, its functional responses were not similar to those of any monkey network. Finally, the medial and lateral prefrontal networks and the default-mode network, spanning portions of the cortex to which substantial evolution-driven reorganizations have been attributed (Ongür et al., 2003), showed the least functional similarities between monkeys and humans.

\section{Discussion}

A basic challenge in comparative neuroscience is to develop comprehensive models explaining topological and functional reorganizations in the brain that have occurred during evolution. Previous studies focused on structural changes, particularly with regard to overall brain size and cortical surface areas of different regions, as well as their microstructure and structural connections (Krubitzer, 2007; Kaas, 2012). In turn, comparative neuroimaging studies mapped brain areas responsible for the same perceptual or cognitive function in different primate species $(\mathrm{Na}-$ kahara et al., 2002; Vanduffel et al., 2002; Orban et al., 2004; Tsao et al., 2008; Pinsk et al., 2009) to evaluate functional correspondences in specific portions of the cortex.

The present study has, for the first time, used a combined topological and functional comparative approach to investigate which, and to what degree, cortical networks may have undergone evolution-driven reorganization. In particular, we evaluated the presence of possible novel networks in humans, which most likely result from evolutionary changes related to cortical surface expansion (Sherwood et al., 2008), and of redeployed networks, which are structurally similar across species but carry out at least partially different functions (Fitch, 2011).

To address this question, we developed an integrated framework in which both topological and functional similarities be- tween monkey and human brains were assessed at the network level rather than at the area level, merging different lines of evidence from previous studies (Orban et al., 2004; Krubitzer, 2007; Van Essen and Dierker, 2007; Allman et al., 2010; Mars et al., 2011). To define monkey and human networks in a taskindependent manner, we used ICA on resting-state fMRI data. This represents an important distinction compared with our previous studies, in which we used ICA only on natural vision data (Mantini et al., 2012a). ICA is a data-driven method that separates independent spatiotemporal patterns of brain activity (Bartels and Zeki, 2005), with no prior knowledge about waveform and/or spatial distribution. Voxels belonging to a given ICA pattern have higher temporal correlations among themselves than with voxels belonging to other patterns. For these reasons, ICA permits us to delineate RSNs in an unbiased manner, and in particular obviates the need to use a seed for functional connectivity analysis (Beckmann et al., 2005). In face of this methodological advantage, a number of limitations should be considered. In resting-state fMRI studies based on ICA, there is uncertainty about the precise number of RSNs that can be identified, the exact topology of the same RSN is not always consistent across experiments, and the full set on RSNs does not cover the whole cortex. Furthermore, the ICA decomposition of fMRI data is designed to detect networks of areas rather than single areas, the investigation of which goes beyond the scope of the present study.

To assess the topological correspondence of RSNs between species, we used a cortical surface expansion model defined on a set of monkey and human landmarks (Van Essen and Dierker, 2007). This model was used by our study to warp the monkey RSNs to human space. It should be acknowledged that its accuracy may vary across the cortex, being less precise at greater distances from landmark regions. We ensured, however, that this 
a
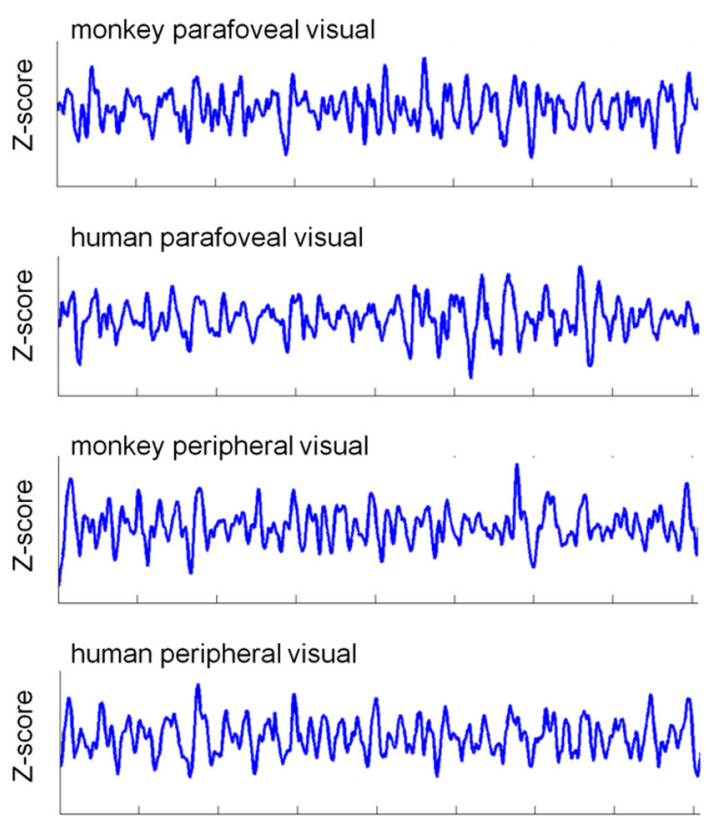

monkey early auditory
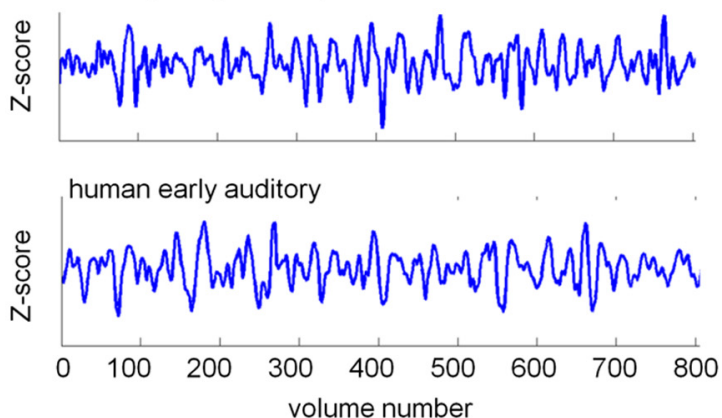

b

canonical correlations

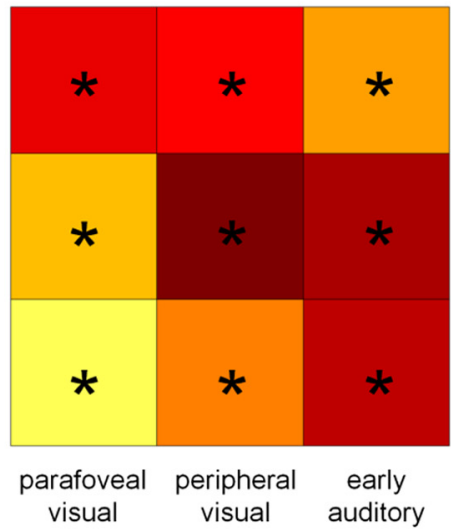

human networks

c

partial correlations

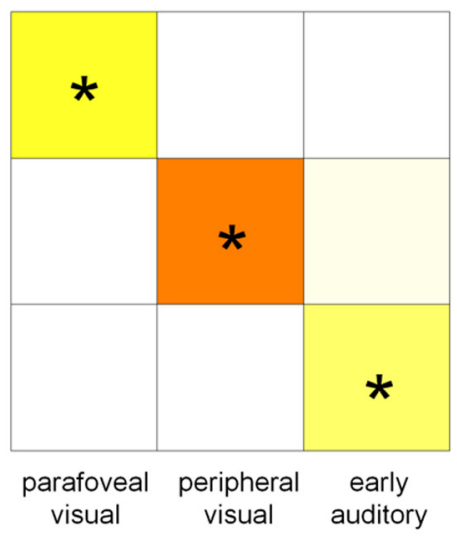

human networks parafoveal

visual

peripheral
visual

early

auditory

0.4

corr

visual

peripheral
visual

early

auditory

Figure 12. Functional responses of monkey and human networks. We have selected three pairs of networks (parafoveal visual, peripheral visual, and early auditory) with relatively large evoked activities in the two species to illustrate in detail the effect of using partial correlations rather than canonical correlations. $\boldsymbol{a}$, Activity time courses of the six networks used as input for the interspecies correlation analysis. $\boldsymbol{b}, \boldsymbol{c}$, Canonical and partial correlations matrices showing the similarities between the network time courses in the two species. Significant values, assessed by means of permutation testing $(p<0.05)$, are marked with stars. It can be noted that the partial correlations are generally smaller then canonical correlations, but likely to be more specific, as significant values are found only between topologically corresponding networks.

variability had no direct impact on our results: the spatial correspondences between monkey and human networks were always well defined (Table 2), i.e., networks in one species never correlated substantially with more than one network in the other species (Figs. 6, 8).

As a final step in our comparative analysis, we used the ISAC approach (Mantini et al., 2012a,b) to assess putative functional similarities between monkey and human networks. We analyzed natural vision fMRI data and we specifically measured the correlation between their sensory-driven responses. It is important to consider that a lack of interspecies functional similarities may result from the fact that the significance of some movie events that drive fMRI activity is different for monkeys and humans (Shepherd et al., 2010). This potential limitation did not prevent us from finding functionally similar responses for several topologically corresponding networks in the two species, but might have led to an underestimation of the interspecies functional correspondences.

Overall, our findings are consistent with the general idea that sensory, motor, and attention capacities are evolutionarily old and generally well developed in monkeys (Corbetta and Shul- man, 2002; Orban et al., 2004; Graziano, 2006; Passingham, 2009). Significantly, we found the human ventral attention network (Corbetta and Shulman, 2002) to have a topological and functional equivalent in the macaque (Figs. $7 a, 13$ ). This result is in line with the existence of an inferior parietal network in the monkey dedicated to attentional reorienting, as thus far suggested only by neurophysiological studies (Steinmetz and Constantinidis, 1995). Notably, the similarity between the dorsal somatomotor networks of the two species and also between dorsal attention networks was lower than we expected. The lack of any significant functional correspondence between the dorsal somatomotor networks (Fig. 5b) of the two species, a network dedicated mainly to the control of foot and lower limbs, may be related to bipedalism in humans but not in monkeys. As for the dorsal attention network, the relatively low functional correspondence between species may partly result from the limited understanding of movie events that monkeys possess compared with humans (Mantini et al., 2012a,b).

Our data are consistent with a parallel progression of cortical expansion and functional redeployment during evolution, and suggest that changes in brain anatomy and function were strongly 


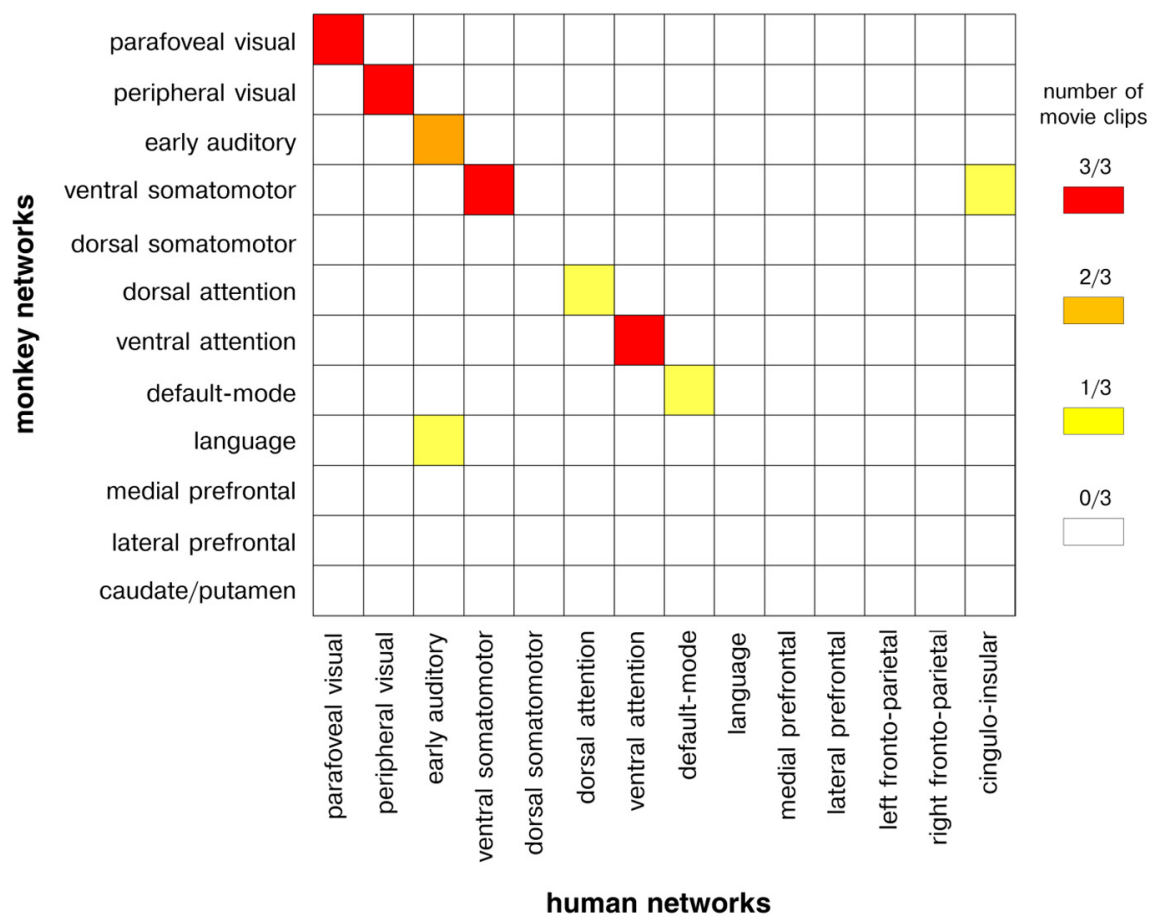

Figure 13. Consistency of significant functional responses between monkey and human networks. Partial correlations were calculated using the ISAC approach on natural vision fMRI data, independently for each movie clip. The three resulting matrices were binarized (threshold based on permutation testing, $p<0.05$ ) and summed to represent the consistency of significant functional responses.

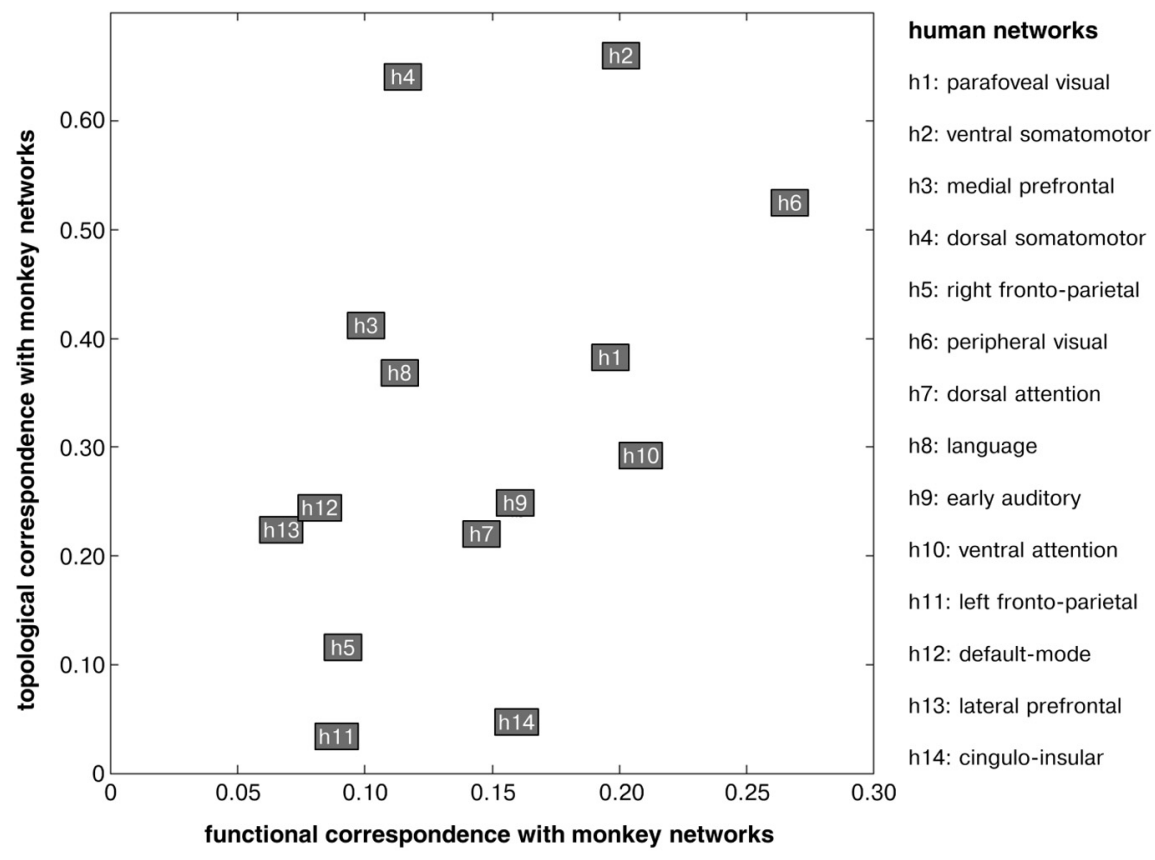

Figure 14. Topological and functional correspondence of human networks compared with monkey networks. These correspondences were measured, for each human network, as the maximum spatial and temporal correlations, respectively, across monkey networks.

coupled. According to the cortical expansion theory, novel networks emerged during primate evolution in specific frontal and parietal regions, where the cortical sheet experienced the greatest enlargement (Sherwood et al., 2008; Van Essen et al., 2012). Our analysis provided evidence for two novel, lateralized frontoparietal networks in humans not found in monkeys (Fig. 9a,b). Clus- ter analysis on RSN maps revealed that the spatial patterns of these networks did not match that of any monkey RSN. Second, the ISAC analyses showed their functional responses in the present natural vision experiment to be species specific. Of course, a negative finding needs to be considered cautiously, since future functional tests might yet reveal similarities between the functional responses of these human networks and networks in the monkey. Other lines of evidence, however, support the concept that the two human frontoparietal networks may be evolutionarily novel. They have been implicated in behavioral control and human intelligence (Duncan et al., 2000), and in various forms of procedural and abstract reasoning in the left and right hemisphere, respectively (Dehaene et al., 2003). These skills are presumably human specific (Passingham, 2009) and are, for example, crucial for tool use (Frey, 2008) and making logic inferences (Dehaene et al., 2003), respectively. Furthermore, they are located in brain regions with the largest cortical surface expansions (Van Essen and Dierker, 2007) and are characterized by different connectional properties compared with the monkey (Mars et al., 2011). These changes may underlie the acquisition of novel cognitive abilities during evolution (Duncan et al., 2000; Dehaene et al., 2003).

The detection of human networks with topological but not functional correspondents in the monkey may lend support to the redeployment theory, according to which pre-existing networks have been repurposed during evolution to carry out new functions (Anderson, 2010; Fitch, 2011). The finding that the neuronal circuitry of the language network (Fig. $7 f$ ) may have been redeployed (Fitch, 2011) is corroborated by the existence in macaques and chimpanzees of precursors of the arcuate fasciculus (Rilling et al., 2008), the white matter fiber tract involved in human language. Also the default-mode network (Fig. 7d) and the medial and lateral prefrontal networks (Fig. $7 b, e$ ) may have been redeployed. For instance, it has been proposed that the default-mode network has expanded its functions in support of spontaneous cognition (Mantini et al., 2011; Pearson et al., 2011; Stawarczyk et al., 2011). However, the possibility should be considered that interspecies differences in functional responses may also be driven by the fact that monkeys are actively rewarded during the experiment while humans are not, or may be due to differences in the perceptions of the movie clips by the two species. Hence, as has been argued above, additional functional tests are warranted to corroborate the present findings. Never- 
theless it is important to note that in the present natural vision experiment, all networks showed significant intersubject correlation within a species (Table 3), which indicates they were all involved in the processing of the stimuli.

In summary, we have compared the spatial structures of brain networks in humans and monkeys and have examined their functional signatures to establish similarities and differences. Importantly, we have provided evidence for cortical networks that were evolutionarily conserved, and for networks putatively involved in topological and functional reorganizations during primate evolution. Our results suggest the coalescence of different reorganization mechanisms, including the functional reuse of preexisting networks and the development of novel networks in cortical regions with the greatest anatomical expansion. Our framework for investigating topological and functional correspondences between cortical networks in primate species, complemented with comparative behavioral studies, may shed light on how human-specific cognitive abilities emerged.

\section{References}

Allman JM, Tetreault NA, Hakeem AY, Manaye KF, Semendeferi K, Erwin JM, Park S, Goubert V, Hof PR (2010) The von Economo neurons in frontoinsular and anterior cingulate cortex in great apes and humans. Brain Struct Funct 214:495-517. CrossRef Medline

Anderson ML (2010) Neural reuse: a fundamental organizational principle of the brain. Behav Brain Sci 33:245-266; discussion 266-313. CrossRef Medline

Bartels A, Zeki S (2005) The chronoarchitecture of the cerebral cortex. Philos Trans R Soc Lond B Biol Sci 360:733-750. CrossRef Medline

Beckmann CF, DeLuca M, Devlin JT, Smith SM (2005) Investigations into resting-state connectivity using independent component analysis. Philos Trans R Soc Lond B Biol Sci 360:1001-1013. CrossRef Medline

Calhoun VD, Adali T, Pearlson GD, Pekar JJ (2001) A method for making group inferences from functional MRI data using independent component analysis. Hum Brain Mapp 14:140-151. CrossRef Medline

Corbetta M, Shulman GL (2002) Control of goal-directed and stimulusdriven attention in the brain. Nat Rev Neurosci 3:201-215. Medline

Damoiseaux JS, Rombouts SA, Barkhof F, Scheltens P, Stam CJ, Smith SM, Beckmann CF (2006) Consistent resting-state networks across healthy subjects. Proc Natl Acad Sci U S A 103:13848-13853. CrossRef Medline

Dehaene S, Piazza M, Pinel P, Cohen L (2003) Three parietal circuits for number processing. Cogn Neuropsychol 20:487-506. CrossRef Medline

Duncan J, Seitz RJ, Kolodny J, Bor D, Herzog H, Ahmed A, Newell FN, Emslie H (2000) A neural basis for general intelligence. Science 289:457-460. CrossRef Medline

Esposito F, Scarabino T, Hyvarinen A, Himberg J, Formisano E, Comani S, Tedeschi G, Goebel R, Seifritz E, Di Salle F (2005) Independent component analysis of fMRI group studies by self-organizing clustering. Neuroimage 25:193-205. CrossRef Medline

Fitch WT (2011) The evolution of syntax: an exaptationist perspective. Front Evol Neurosci 3:9. Medline

Fox MD, Raichle ME (2007) Spontaneous fluctuations in brain activity observed with functional magnetic resonance imaging. Nat Rev Neurosci 8:700-711. CrossRef Medline

Frey SH (2008) Tool use, communicative gesture and cerebral asymmetries in the modern human brain. Philos Trans R Soc Lond B Biol Sci 363: 1951-1957. CrossRef Medline

Graziano M (2006) The organization of behavioral repertoire in motor cortex. Annu Rev Neurosci 29:105-134. CrossRef Medline

Hasson U, Nir Y, Levy I, Fuhrmann G, Malach R (2004) Intersubject synchronization of cortical activity during natural vision. Science 303:16341640. CrossRef Medline

Hutchison RM, Leung LS, Mirsattari SM, Gati JS, Menon RS, Everling S (2011) Resting-state networks in the macaque at 7T. Neuroimage 56: 1546-1555. CrossRef Medline

Joly O, Pallier C, Ramus F, Pressnitzer D, Vanduffel W, Orban GA (2012) Processing of vocalizations in humans and monkeys: a comparative fMRI study. Neuroimage 62:1376-1389. CrossRef Medline

Kaas JH (2012) The evolution of neocortex in primates. Prog Brain Res 195:91-102. CrossRef Medline
Kolster H, Mandeville JB, Arsenault JT, Ekstrom LB, Wald LL, Vanduffel W (2009) Visual field map clusters in macaque extrastriate visual cortex. J Neurosci 29:7031-7039. CrossRef Medline

Kolster H, Peeters R, Orban GA (2010) The retinotopic organization of the human middle temporal area MT/V5 and its cortical neighbors. J Neurosci 30:9801-9820. CrossRef Medline

Krubitzer L (2007) The magnificent compromise: cortical field evolution in mammals. Neuron 56:201-208. CrossRef Medline

Laird AR, Eickhoff SB, Li K, Robin DA, Glahn DC, Fox PT (2009) Investigating the functional heterogeneity of the default mode network using coordinate-based meta-analytic modeling. J Neurosci 29:14496-14505. CrossRef Medline

Lowe MJ, Mock BJ, Sorenson JA (1998) Functional connectivity in single and multislice echoplanar imaging using resting-state fluctuations. Neuroimage 7:119-132. CrossRef Medline

Mantini D, Perrucci MG, Del Gratta C, Romani GL, Corbetta M (2007) Electrophysiological signatures of resting state networks in the human brain. Proc Natl Acad Sci U S A 104:13170-13175. CrossRef Medline

Mantini D, Gerits A, Nelissen K, Durand JB, Joly O, Simone L, Sawamura H, Wardak C, Orban GA, Buckner RL, Vanduffel W (2011) Default mode of brain function in monkeys. J Neurosci 31:12954-12962. CrossRef Medline

Mantini D, Corbetta M, Romani GL, Orban GA, Vanduffel W (2012a) Data-driven analysis of analogous brain networks in monkeys and humans during natural vision. Neuroimage 63:1107-1118. CrossRef Medline

Mantini D, Hasson U, Betti V, Perrucci MG, Romani GL, Corbetta M, Orban GA, Vanduffel W (2012b) Interspecies activity correlations reveal functional correspondence between monkey and human brain areas. Nat Methods 9:277-282. CrossRef Medline

Mars RB, Jbabdi S, Sallet J, O’Reilly JX, Croxson PL, Olivier E, Noonan MP, Bergmann C, Mitchell AS, Baxter MG, Behrens TE, Johansen-Berg H, Tomassini V, Miller KL, Rushworth MF (2011) Diffusion-weighted imaging tractography-based parcellation of the human parietal cortex and comparison with human and macaque resting-state functional connectivity. J Neurosci 31: 4087-4100. CrossRef Medline

Moeller S, Nallasamy N, Tsao DY, Freiwald WA (2009) Functional connectivity of the macaque brain across stimulus and arousal states. J Neurosci 29:5897-5909. CrossRef Medline

Nakahara K, Hayashi T, Konishi S, Miyashita Y (2002) Functional MRI of macaque monkeys performing a cognitive set-shifting task. Science 295: 1532-1536. CrossRef Medline

Ongür D, Ferry AT, Price JL (2003) Architectonic subdivision of the human orbital and medial prefrontal cortex. J Comp Neurol 460:425-449. CrossRef Medline

Orban GA, Van Essen D, Vanduffel W (2004) Comparative mapping of higher visual areas in monkeys and humans. Trends Cogn Sci 8:315-324. CrossRef Medline

Passingham R (2009) How good is the macaque monkey model of the human brain? Curr Opin Neurobiol 19:6-11. CrossRef Medline

Pearson JM, Heilbronner SR, Barack DL, Hayden BY, Platt ML (2011) Posterior cingulate cortex: adapting behavior to a changing world. Trends Cogn Sci 15:143-151. CrossRef Medline

Pinsk MA, Arcaro M, Weiner KS, Kalkus JF, Inati SJ, Gross CG, Kastner S (2009) Neural representations of faces and body parts in macaque and human cortex: a comparative FMRI study. J Neurophysiol 101:2581-2600. Medline

Price CJ (2000) The anatomy of language: contributions from functional neuroimaging. J Anat 197:335-359. CrossRef Medline

Rakic P (1995) A small step for the cell, a giant leap for mankind: a hypothesis of neocortical expansion during evolution. Trends Neurosci 18:383-388. CrossRef Medline

Rilling JK, Glasser MF, Preuss TM, Ma X, Zhao T, Hu X, Behrens TE (2008) The evolution of the arcuate fasciculus revealed with comparative DTI. Nat Neurosci 11:426-428. CrossRef Medline

Rushworth MF, Noonan MP, Boorman ED, Walton ME, Behrens TE (2011) Frontal cortex and reward-guided learning and decision-making. Neuron 70:1054-1069. CrossRef Medline

Schreiber T, Schmitz A (1996) Improved surrogate data for nonlinearity tests. Phys Rev Lett 77:635-638. CrossRef Medline

Shepherd SV, Steckenfinger SA, Hasson U, Ghazanfar AA (2010) Humanmonkey gaze correlations reveal convergent and divergent patterns of movie viewing. Curr Biol 20:649-656. CrossRef Medline

Sherwood CC, Subiaul F, Zawidzki TW (2008) A natural history of the hu- 
man mind: tracing evolutionary changes in brain and cognition. J Anat 212:426-454. CrossRef Medline

Skudlarski P, Jagannathan K, Calhoun VD, Hampson M, Skudlarska BA, Pearlson G (2008) Measuring brain connectivity: diffusion tensor imaging validates resting state temporal correlations. Neuroimage 43:554-561. CrossRef Medline

Smith SM, Fox PT, Miller KL, Glahn DC, Fox PM, Mackay CE, Filippini N, Watkins KE, Toro R, Laird AR, Beckmann CF (2009) Correspondence of the brain's functional architecture during activation and rest. Proc Natl Acad Sci U S A 106:13040-13045. CrossRef Medline

Stawarczyk D, Majerus S, Maquet P, D’Argembeau A (2011) Neural correlates of ongoing conscious experience: both task-unrelatedness and stimulus-independence are related to default network activity. PLoS One 6:e16997. CrossRef Medline

Steinmetz MA, Constantinidis C (1995) Neurophysiological evidence for a role of posterior parietal cortex in redirecting visual attention. Cereb Cortex 5:448-456. CrossRef Medline

Striedter GF (2002) Brain homology and function: an uneasy alliance. Brain Res Bull 57:239-242. CrossRef Medline

Tsao DY, Moeller S, Freiwald WA (2008) Comparing face patch systems in macaques and humans. Proc Natl Acad Sci U S A 105:19514-19519. CrossRef Medline

van den Heuvel MP, Mandl RC, Kahn RS, Hulshoff Pol HE (2009) Func- tionally linked resting-state networks reflect the underlying structural connectivity architecture of the human brain. Hum Brain Mapp 30:31273141. CrossRef Medline

Van Essen DC (2012) Cortical cartography and Caret software. Neuroimage 62:757-764. CrossRef Medline

Van Essen DC, Dierker DL (2007) Surface-based and probabilistic atlases of primate cerebral cortex. Neuron 56:209-225. CrossRef Medline

Van Essen DC, Glasser MF, Dierker DL, Harwell J, Coalson T (2012) Parcellations and hemispheric asymmetries of human cerebral cortex analyzed on surface-based atlases. Cereb Cortex 22:2241-2262. CrossRef Medline

Vanduffel W, Fize D, Mandeville JB, Nelissen K, Van Hecke P, Rosen BR, Tootell RB, Orban GA (2001) Visual motion processing investigated using contrast agent-enhanced fMRI in awake behaving monkeys. Neuron 32:565-577. CrossRef Medline

Vanduffel W, Fize D, Peuskens H, Denys K, Sunaert S, Todd JT, Orban GA (2002) Extracting 3D from motion: differences in human and monkey intraparietal cortex. Science 298:413-415. CrossRef Medline

Vincent JL, Patel GH, Fox MD, Snyder AZ, Baker JT, Van Essen DC, Zempel JM, Snyder LH, Corbetta M, Raichle ME (2007) Intrinsic functional architecture in the anaesthetized monkey brain. Nature 447:83-86. CrossRef Medline 